\title{
MULTI-TIER MULTI-TENANT NETWORK SLICING: A MULTI-DOMAIN GAMES APPROACH
}

\author{
Sunday Oladayo Oladejo $^{1}$, Stephen Obono Ekwe ${ }^{1}$, Lateef Adesola Akinyemi ${ }^{1,2}$ \\ ${ }^{1}$ Department of Electrical Engineering, University of Cape Town, Cape Town, South Africa, ${ }^{2}$ Department of Electronic and \\ Computer Engineering, Lagos State University, Epe, Lagos State, Nigeria
}

NOTE: Corresponding author: Sunday Oladayo Oladejo, oldsun002@myuct.ac.za

\begin{abstract}
The $5 G$ slice networks will play a critical role in meeting the stringent quality-of-service requirements of different use cases, reducing the Capital Expenditure (CapEX) and Operational Expenditure (OpEX) of mobile network operators. Owing to the flexibility and ability of $5 \mathrm{G}$ slice networks to meet the needs of different verticals, it attracts new network players and entities to the mobile network ecosystem, and therefore it creates new business models and structures. Motivated by this development, this paper addresses the dynamic resource allocation in a multi-slice multi-tier multi-domain network with different network players. The dynamic resource allocation problem is formulated as a maximum utility optimisation problem from a multiplayer multi-domain perspective. Furthermore, a 3-level hierarchical business model comprising Infrastructure Providers (InPs), Mobile Virtual Network Operators (MVNOs), Service Providers (SPs), and slice users are investigated. We propose two schemes: a multi-tier multi-domain slice user matching game scheme and a distributed backtracking multiplayer multi-domain game scheme in solving the transformed maximum utility optimisation problem. We compare the multi-tier multi-tenant multi-domain game scheme with a Genetic Algorithm (GA) Intelligent Latency-Aware Resource (GI-LARE) allocation scheme, and a static slicing resource allocation scheme via Monte Carlo simulation. Our findings reveal that the proposed scheme significantly outperforms these other schemes.
\end{abstract}

Keywords - 5G, multi-domain, multi-tenancy, MVNO, network slicing, resource allocation, SP

\section{INTRODUCTION}

The Network Slicing (NS) paradigm entails the abstraction of the physical mobile network and its hard resources into logical autonomous networks via network softwarisation technology [1]. To this end, it has been widely acknowledged that NS will greatly improve the resource utilisation of the mobile networks and significantly reduces their Capital Expenditure (CapEx) and Operational Expenditure (OpEx). Consequently, efforts have been geared towards achieving these goals through diverse resource management frameworks, owing to complex business models of NS. However, with the flexible and agile characterisation of NS architecture, its business model departs radically from the traditional models $[2,3,4]$. Hence, new mobile entities such as Infrastructure Providers (InPs), Mobile Virtual Network Operators (MVNOs), Over-the-top Providers (OTTs), Content Providers (CPs), Service Providers (SPs), Backhaul Operators (BOs), and Vehicular-Content Providers (VCPs) have become critical industry players.

To exploit the capabilities of $5 \mathrm{G}$ and beyond mobile networks, the self-interests of the diverse network players would have to be efficiently managed. To this end, the challenge of managing network resources to achieve optimal network performance and economic advantages to the network players is non-trivial. This paper examines the resource allocation bottleneck in a Multi-Tier multiTenant multi-Slice multi-Domain (M-TTSD) 5G and beyond network $[5,6]$. Specifically, we consider an M-TTSD $5 \mathrm{G}$ network model with a business model consisting of SP, MVNO, InP, and slice users.
The multi-domain concept for NS refers to the pooling of network resources from multiple InPs which are then virtualised and sliced to meet the needs of slice users and diverse verticals [7]. Verticals are business and public entities that run their service applications on mobile networks. Instances of verticals are defence agencies, transport systems, health institutions, power and energy utilities, disaster and emergency agencies, manufacturing, and agricultural chains. The importance of multidomain NS is emphasised in resource aggregation, coverage area extension, reliability of services, and the opportunity to further make the business model Incentive Compatible (IC) for network players $[8,9]$. On the other hand, in the single domain model, the NS resources are primarily from the virtualised resources of only one InP. A vast corpus of literature on NS has focused on the single domain model despite its non-flexibility and limited resource constraints which make it unsuitable for the future mobile networks [8, 10]. In Fig. 1(a)-(c), we illustrate the traditional, single, and multi-domains multi-tenant NS paradigms. This work focuses on the M-TTSD network. In a multi-domain system, several InPs provide infrastructure and network resources services, and to this end, the infrastructure and network resources owned by an InP represent a network domain. The multi-tenancy paradigm entails several independent MVNOs (i.e., otherwise known as tenants) share the same physical infrastructure and network resources owned by an InP. The term "tenants" may be extended to SP depending on the 


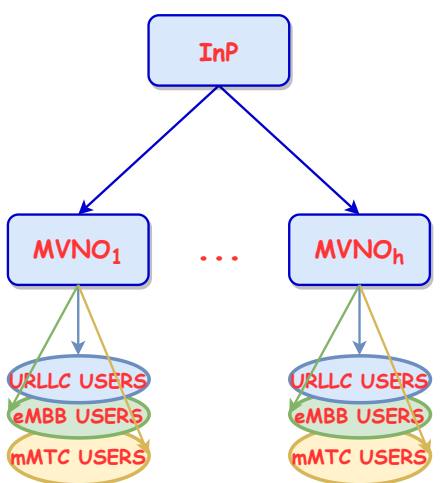

(a) Traditional Multi-Tenancy

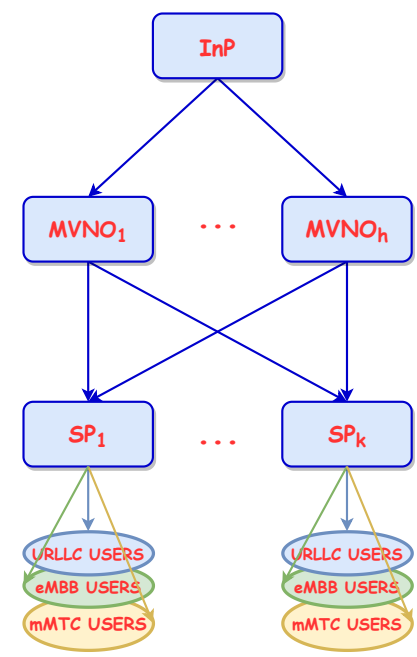

(b) Single-Domain Multi-Tenant NS

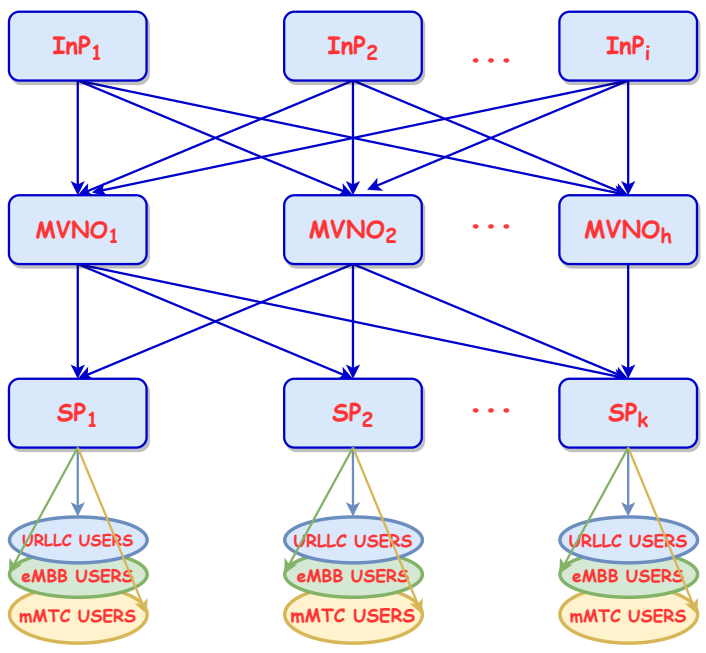

(c) Multi-Domain Multi-Tenant NS

Fig. 1 - Illustration of the domain type of network slicing.

flexibility of the network architecture. In an M-TTSD network, an SP satisfies the demands of slice users by bidding for virtual resources from multiple MVNOs. Similarly, an MVNO bids for mobile network resources from multiple InPs to meet the demands of SPs associated with it. The M-TTSD network explores the benefits and possibilities of tenants connecting to several network domains for coverage extension, scalability, and network resource optimisation.

Efficient resource management is pivotal to the optimal operation of the M-TTSD network $[5,6]$. Moreover, static slicing and centralised resource management frameworks would not be practicable in M-TTSD networks. To fully exploit the flexibility and dynamic characterisation of the slice traffic, it is important to note that not adequately addressing the resource allocation challenge in an M-TTSD network will adversely affect the Quality-of-Service (QoS) of slice users and the IC of network players, thereby jeopardising the sustainability of $5 \mathrm{G}$ slice networks and future networks.

The main contributions of this work can be summarised as follows.

\subsection{Contributions}

The main contributions of this work can be summarised as follows.

1. We consider a latency-aware dynamic resource allocation framework for an M-TTSD network with enhanced Mobile Broadband (eMBB), massive Machine-Type Communications (mMTC), and UltraReliable Low-Latency Communications (URLLC) slice users, respectively. The framework allocates resources to slice users by engaging network parameters (such as packet size, packet arrival rate, packet loss probability, delay bound, slice users distribution, cell load, tier load, and bidding budgets of network entities such as MVNO and SP) and slice users' parameters (such as slice users' location, slice use case QoS requirement, associated interference).

2. Other than the traditional two-player network approach, we consider a multi-tenant multi-domain network with entities comprising InPs, MVNOs, and SPs, respectively. To this end, a three-stage multidomain auction game based on the Fisher Market (FM) principle and shared-constrained proportionality is exploited to facilitate an agile and dynamic business model for $5 \mathrm{G}$ NS and beyond networks; and also to maximise the utility of the respective players. The InPs, MVNOs, and SPs trade network resources to meet the demands of slice users in a manner that ICs and Individual Rationality (IR) are not compromised.

3. We formulate the slice users' service selection problem in an M-TTSD network as a maximisation problem. To reduce the complexity involved in solving the formulated problem, a hierarchical decomposition technique is employed. We develop a multistage matching-theory inspired scheme to optimally associate slice users to SPs, MVNOs, and InPs, respectively, in an M-TTSD network. The multistage matching algorithm considers the Signal-to-Interferenceplus-Noise Ratio (SINR) of the slice users, slice QoS requirements in the course of matching slice users to the respective network tiers and InP.

4. We develop a distributed backtracking algorithm that aids buyers and sellers in the respective stages to trade network resources in an incomplete information scenario. The backtracking algorithm takes 
into consideration bidders preferences, bids, budgets, and the demand at the seller's side in the auction games.

5. Finally, we present the performance evaluation of the proposed framework via extensive Monte Carlo simulations. The proposed framework is compared with schemes in the literature such as a genetic algorithm-based dynamic resource allocation (GILARE) and the non-dynamic approach of StaticSlicing (SS) schemes.

\subsection{Article organisation}

The rest of the article is structured as follows. We devote Section 2 to the discussion of related literature. In Section 3, we focus on the system model. Section 4 presents the multi-domain multi-tenant auction games framework. Further, Section 5 describes the resource allocation framework in an M-TTSD 5G NS network. Then in Section 6, we formulate the M-TTSD resource allocation problem. Section 7 details the reformulation steps and proposed solutions. In Section 8, the complexity analysis of the proposed solutions is discussed. Then, in Section 9, we present the numerical results of the performance evaluation. Finally, conclusions are drawn in Section 10.

\section{RELATED WORK}

Our work builds on the Fisher Market (FM), which is one of the most widely known models in mathematical economics $[11,12]$. In FM, a set of buyers with limited budgets (of no intrinsic value) to purchase divisible goods from a set of sellers with the sole aim of maximising their respective non-negative utility $[13,14,15]$. In the same vein, we liken InPs, MVNOs, SPs, and slice users to sellers and buyers in the M-TTSD network.

The works in $[16,17,18,19]$ proposed optimisation frameworks for maximising the utility of slice users in a single-domain multi-tenant network. The proposed frameworks employed static-slicing schemes. Network economics variables such as the budgets of the respective network players were not considered.

Contrary to the aforementioned works in $[16,17,18,19]$, the authors [20, 21, 22, 23] adopted the network economics variables (such as budget, auctions) in their proposed schemes for efficient resource allocation. Auction game-based schemes were proposed for resource allocation between an InP (seller) and MVNOs (buyers).

In [24], the authors proposed a dynamic virtual resource allocation framework premised on the widely known biological population model called Lotka-Volterra [46]. The virtual resources of the multiple InPs are centralised and then allocated to MVNOs. Similar to [24], the authors in $[25,26,27]$ proposed dynamic resource allocation schemes which are centrally controlled in heterogeneous networks.

A two-stage resource allocation scheme for a multi-tenant slice network was proposed in [28] to address the differ- ent levels of granularity of resources in InP-MVNO and MVNO-slice users stages. Additionally, the authors examined the transport and core networks of the $5 \mathrm{G}$ network. Similar to [28], an end-to-end 5G NS was examined in [29]. The authors proposed a two-level centralised resource allocation scheme termed as Uppertier First with Latency-bounded over-provision Prevention (UFLOP). The UFLOP prevents the overprovisioning of resources between the InP and MVNOs while meeting the MVNOs' Service Level Agreement (SLA) and slice use cases' latency requirements.

In $[30,31]$, the authors categorised slice users into Guaranteed Bit Rate (GBR) and non-Guaranteed Bit Rate (nonGBR) users. In [30], the authors proposed a Markovbased resource allocation for a multi-slice multi-tenant network. The proposed Markov-based algorithm was able to characterise and model the stochastic behaviour of slice use case requirements and channel behaviour. Moreover, the authors [31] proposed an MVNO virtual resource allocation architecture to accurately predict the bandwidth requirement of different slice use cases, respectively. The proposed architecture relies on the Long Short-Term Memory (LSTM) neural network for prediction.

An iterative double-auction mechanism for a multi-tenant multi-domain SDN-based network was proposed by the authors in [32] to maximise the utility of the respective MVNOs.

Whereas the authors in [7] enumerated different business models for wireless network virtualisation in $5 \mathrm{G}$ and beyond era. Additionally, the authors did not consider the Radio Access Network (RAN) and multi-slice characterisation of the network with its diverse QoS requirement. In $[33,34,35]$, a two-level stackelberg game and winner determination models for dynamic pricing were proposed by the authors. The authors considered a traditional multi-tenancy scenario with a single seller (InP) and multiple buyers (MVNOs). In [34], a hierarchical auction model was examined. In [36], the authors considered a hierarchical multi-tenant network comprising InPs, an MVNO, and SPs. The scenario is such that an SP can only bid for resources from one MVNO, while the MVNO can network resources from multiple InPs.

Similar to [36], the authors in [37] adapted the NonOrthogonal Multiple Access (NOMA) technique to allocate resources of the MVNOs and SPs. A Tchebycheff technique was employed in solving the Multi-Objective resource allocation Optimisation Problem (MOOP). The authors in $[38,39]$ employed a MOOP technique to the single domain heterogeneous networks.

A deep learning-based caching and leasing framework was proposed in [40] for a traditional single-domain multi-tenant network comprising an InP and a set of MVNOs. The algorithm predicts the resource leasing patterns of respective MVNOs for profit maximisation. In $[41,42]$, the authors proposed a weighted proportional allocation scheme that allocates power in a single-tier traditional multi-tenancy virtual network. A bidding strat- 
Table 1 - Related Works Summary (OYes $\bigcirc$ No OPartial)

\begin{tabular}{|c|c|c|c|c|c|c|c|c|c|c|c|c|c|}
\hline Literature & $\begin{array}{l}\text { NS } \\
\text { Use } \\
\text { Case }\end{array}$ & $\begin{array}{l}\text { SS Al- } \\
\text { loc. }\end{array}$ & $\begin{array}{l}\text { Dyn. } \\
\text { Alloc. }\end{array}$ & \begin{tabular}{l|} 
Dist. \\
Ntwk
\end{tabular} & $\begin{array}{l}\text { Cen. } \\
\text { Ntwk }\end{array}$ & MVNO & SP & InP & $\begin{array}{l}\text { Single } \\
\text { Do- } \\
\text { main } \\
\text { Ntwk }\end{array}$ & $\begin{array}{l}\text { Multi- } \\
\text { Domain } \\
\text { Ntwk }\end{array}$ & $\begin{array}{l}\text { Multi- } \\
\text { Tier } \\
\text { Ntwk }\end{array}$ & $\begin{array}{l}\text { Single } \\
\text { Tier } \\
\text { Ntwk }\end{array}$ & $\begin{array}{l}\text { Economic } \\
\text { Var. }\end{array}$ \\
\hline$[16,17]$ & $\bullet$ & $\bullet$ & 0 & 0 & 0 & 0 & 0 & $\bullet$ & $\bullet$ & 0 & 0 & 0 & 0 \\
\hline [18] & $\bullet$ & $\bullet$ & 0 & 0 & 0 & 0 & 0 & 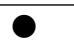 & $\bullet$ & 0 & 0 & 0 & D \\
\hline [19] & 0 & 0 & - & $\bullet$ & 0 & $\bullet$ & 0 & - & - & 0 & $\bullet$ & 0 & 0 \\
\hline $\begin{array}{l}{[20,21} \\
22,23]\end{array}$ & O & O & $\bullet$ & $\bullet$ & O & $\bullet$ & 0 & $\bullet$ & $\bullet$ & O & O & $\bullet$ & 0 \\
\hline [24] & 0 & 0 & - & 0 & 0 & 0 & 0 & - & 0 & - & 0 & 0 & 0 \\
\hline [25] & 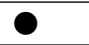 & 0 & - & 0 & 0 & 0 & 0 & 0 & - & 0 & 0 & 0 & 0 \\
\hline [26] & 0 & 0 & - & 0 & 0 & 0 & 0 & 0 & 0 & 0 & 0 & 0 & 0 \\
\hline [27] & $\bullet$ & 0 & $\bullet$ & 0 & 0 & 0 & 0 & $\bullet$ & $\bullet$ & 0 & 0 & 0 & 0 \\
\hline [28] & $\bullet$ & 0 & $\bullet$ & 0 & $\bullet$ & $\bullet$ & 0 & $\bullet$ & $\bullet$ & 0 & $\bullet$ & 0 & 0 \\
\hline [29] & - & 0 & - & 0 & $\bullet$ & $\bullet$ & 0 & - & 0 & $\bullet$ & 0 & $\bullet$ & 0 \\
\hline [30] & D & 0 & - & 0 & $\bullet$ & $\bullet$ & 0 & - & - & 0 & 0 & $\bullet$ & 0 \\
\hline [31] & $\bullet$ & $\circ$ & $\bullet$ & 0 & $\bullet$ & 0 & 0 & $\bullet$ & $\bullet$ & 0 & 0 & 0 & D \\
\hline [32] & 0 & 0 & - & 0 & 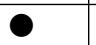 & 0 & 0 & $\bullet$ & 0 & $\bullet$ & 0 & 0 & $\bullet$ \\
\hline [7] & 0 & O & - & 0 & 0 & 0 & 0 & - & O & $\bullet$ & 0 & 0 & $\bullet$ \\
\hline [33] & 0 & $\bullet$ & 0 & 0 & $\bullet$ & $\bullet$ & 0 & 0 & $\bullet$ & 0 & 0 & 0 & $\bullet$ \\
\hline [34] & 0 & $\bullet$ & 0 & 0 & $\bullet$ & $\bullet$ & 0 & $\bullet$ & 0 & 0 & 0 & 0 & $\bullet$ \\
\hline [35] & 0 & 0 & - & 0 & $\bullet$ & $\bullet$ & 0 & - & - & 0 & 0 & $\bullet$ & - \\
\hline [36] & 0 & 0 & - & 0 & 0 & 0 & - & - & 0 & - & 0 & 0 & - \\
\hline [37] & O & 0 & - & 0 & 0 & 0 & - & - & 0 & - & 0 & 0 & O \\
\hline$[38,39]$ & 0 & $\bullet$ & 0 & 0 & $\bullet$ & 0 & 0 & - & $\bullet$ & 0 & $\bullet$ & 0 & 0 \\
\hline [40] & 0 & 0 & - & 0 & $\bullet$ & $\bullet$ & 0 & - & - & 0 & 0 & 0 & D \\
\hline$[41,42]$ & 0 & $\bullet$ & 0 & 0 & $\bullet$ & $\bullet$ & 0 & $\bullet$ & $\bullet$ & 0 & 0 & 0 & D \\
\hline [43] & 0 & $\bullet$ & 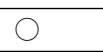 & 0 & 0 & 0 & 0 & $\bullet$ & $\bullet$ & 0 & 0 & 0 & $\bullet$ \\
\hline [44] & $\circ$ & $\bullet$ & 0 & 0 & 0 & 0 & 0 & $\bullet$ & $\bullet$ & 0 & D & 0 & $\circ$ \\
\hline [45] & - & - & 0 & 0 & 0 & 0 & 0 & 0 & - & 0 & 0 & 0 & - \\
\hline
\end{tabular}

egy was employed by the InP for efficient resource allocation. The authors in [43] improved the works in [41, 42] by proposing a Generalised Kelly Mechanism (GKM) for resource trading between an InP and respective MVNOs. Furthermore, the authors in [41, 42, 43] proposed [44] a joint radio resource allocation scheme based on a block upper-bound minimisation algorithm in a heterogeneous virtualised network.

In [45], the author proposed slice admission control premised on network tenants' patience and waiting time characterisation in a multiservice multi-tenant slice network.

We give a detailed summary of the reviewed literature in Table 1. The motivation of this paper stems from the fact that very few works have examined the resource allocation challenge in an M-TTSD network coupled with considerations of the NS use cases and economic constraints of respective network players. We aim to bridge this gap in literature.

\section{SYSTEM MODEL}

In this section, we describe the system model of the MTTSD 5G NS. A pictorial representation of the system model is illustrated in Fig. 2.

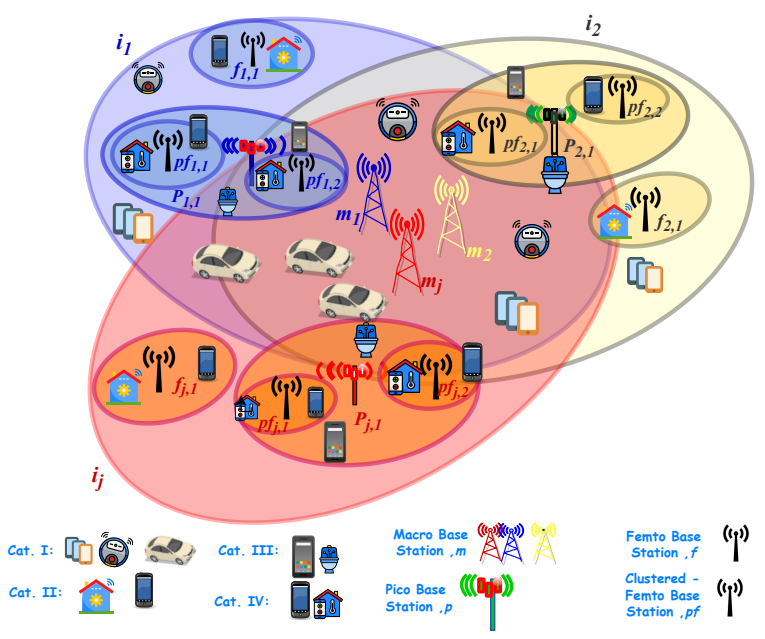

Fig. 2 - Illustration of the system model. 


\subsection{Network players}

Herein, a set of InP numbered as $\mathcal{J}=\{i \mid i \in \mathcal{N}, 1 \leq i \leq$ $|\mathcal{J}|\}$, with unique and independent resources; that is, $i \neq$ $i^{\prime}$, provide services to a set of MVNOs $\mathcal{V}$. Where the set of MVNOs $\mathcal{V}$ is indexed as $\mathcal{V}=\{v|v \in \mathcal{N}, 1 \leq v \leq| \mathcal{V} \mid\}$. An MVNO $v$ is independent and manages its own acquired or leased resources; that is, $v \neq v^{\prime}$, offers virtual services to service providers. Similarly, the set of service providers is numbered as $\mathcal{S}=\{s|s \in \mathcal{N}, 1 \leq s \leq| \mathcal{S} \mid\}$. Following the assumptions for InPs and MVNOs, it is given that $s \neq s^{\prime}$. A service provider $s$ offer a set of slice use-case services denoted $\mathcal{U} \mathcal{C}_{S}$ to subscribers. Herein, $\mathcal{U C} \mathcal{C}_{S}=\{\mathcal{E} \cup \mathcal{M} \cup \mathcal{R}\}$, where $\mathcal{E}, \mathcal{M}$, and $\mathcal{R}$ denote the eMBB, MMTC, and URLLC slice use cases, respectively.

\subsection{Physical network}

Additionally, we examine the physical network of the MTTSD network. In this work, the multi-tier network consists of macro-cells, picocells, clustered femtocells, nonclustered femtocells, and a Vehicle-to-everything (V2X) tier whose resources are owned and managed by the respective InPs. Furthermore, we index the tiers by proceeding with macro-cells. The set of macro-cells owned by $\operatorname{InP} i$ is indexed as $M_{i}=\left\{m_{i}\left|m_{i} \in \mathcal{N}, 1 \leq m_{i} \leq\right| M_{i} \mid\right\}$. Moreover, the set of non-clustered femtocells owned by an InP $i$ is denoted as $\mathcal{F}_{i}=\left\{f_{i}\left|f_{i} \in \mathcal{N}, 1 \leq f_{i} \leq\right| \mathcal{F}_{i} \mid\right\}$. The set of picocells owned by InP $i$ is numbered as $\mathcal{P}_{i}=$ $\left\{p_{i}\left|p_{i} \in \mathcal{N}, 1 \leq p_{i} \leq\right| \mathcal{P}_{i} \mid\right\}$. Besides, a cluster of a femtocells is under the coverage area of a picocell and therefore, the number of clusters is equal to $\left|\mathcal{P}_{i}\right|$. Finally, we define the V2X tier by categorising this tier into two: (i) vehicles engaged in Vehicle-to-Infrastructure (V2I) communication layer, and (ii) those engaged in Vehicle-to-Vehicle (V2V) communication layer [47, 48]. Furthermore, it is important to note that in this paper, we identify users or vehicles in the V2X tier as URLLC users owing to the peculiarities of V2X communications [49, 50, 51, 52]. Moreover, we give a detailed explanation of the V2X layer in Subsection 3.4.

\subsection{Slice-user categorisation}

In this subsection, the slice users in the M-TTSD network are categorised primarily according to their slice use-case type, slice users' SP, and the location of the slice user in the M-TTSD network. Therefore, the categorisation is as follows.

1. Cat. I: The set of eMBB, mMTC, and URLLC users subscribed to an SP $s \in \mathcal{S}$ under the coverage of a macrotier $m_{i} \in M_{i}$ belonging to an InP $i \in \mathcal{J}$ are denoted by $\mathcal{E}_{s, m, i}, \mathcal{M}_{s, m, i}$, and $\mathcal{R}_{s, m, i}$.

2. Cat. II: The set of eMBB and mMTC slice users subscribed to SP $s \in \mathcal{S}$ under the coverage of a nonclustered femtocell $f_{i} \in \mathcal{F}_{i}$ managed by an InP $i \in \mathcal{J}$ are denoted by $\mathcal{E}_{s, f_{i}, i}$ and $\mathcal{M}_{s, f_{i}, i}$.
3. Cat. III: Similarly, the set of slice users requesting for eMBB, and mMTC slices subscribed to SP $s \in \mathcal{S}$ and under the coverage of a picocell $p_{i} \in \mathcal{P}_{i}$ are indexed by $\mathcal{E}_{s, p_{i}, i}$ and $\mathcal{M}_{s, p_{i}, i}$.

4. Cat. IV: Lastly, slice users under the coverage of a clustered femtocell $p f_{i} \in \mathcal{P} \mathcal{F}_{i}$ and subscribed to SP $s \in \mathcal{S}$, are denoted by $\mathcal{E}_{s, p f_{i}, i}$ and $\mathcal{M}_{s, p f_{i}, i}$.

\subsection{V2X communication model}

We model slice users in the V2X-tier as having similar URLLC QoS requirements due to their uniqueness [49, $50,51,52]$. Two widely known approaches to V2Xcommunications are namely [53, 54]: (1) Cellular-V2X (C-V2X), and (2) Dedicated Short Range Communications (DSRC) which is premised on the IEEE 802:11:P standard. Owing to the growing popularity of C-V2X in the communications and automobile industry and coupled with other numerous reasons discussed in $[55,56,57,58]$, we adopt the C-V2X approach in modelling C-V2X users in this work. The set of paired vehicles in the V2V communication layer is indexed as $\mathcal{W}=\{w|w \in \mathcal{N}, 1 \leq w \leq| \mathcal{W} \mid\}$. The PC5 sidelink [55] is employed in the communication of paired vehicles in the V2V layer. Additionally, the set of vehicles in the V2I layer (otherwise known as V2N) is numbered as $\mathcal{R}=\{r|r \in \mathcal{N}, 1 \leq r \leq| \mathcal{R} \mid\}$. These vehicles are connected to macro-base-stations alone to reduce the handover signalling overhead, owing to the large coverage area of the macro-base-stations [59].

\subsection{Channel model}

This paper focuses on the downlink of the M-TTSD network. Specifically, we employ the link-layer model described in $[60,61]$, and the mobility characterisation of slice users. To this end, the channel modelling stage is categorised, respectively, into two: (i) static or moderately mobile slice users, and; (ii) highly mobile slice users. Without loss of generality, eMBB and mMTC slice users are assumed to be static or moderately mobile, while URLLC slice users are categorised as highly mobile.

\subsubsection{Static slice users}

The channel modelling for static slice users $u_{s, i} \in\{\mathcal{E} \cup$ $\mathcal{M}\}$ is dependent on large-scale fading components such as path loss, shadow fading, and the tier in consideration. The propagation model is expressed as [62]:

$$
\lambda_{u, s, j, i}=\Lambda_{j}+35 \log \left(\mathrm{d}_{u, s, j, i}\right)
$$

where $\mathrm{d}_{u, s, j, i}$ is the distance of a slice user $u_{s, i}$ from an access point $j$ belonging to InP $i . \Lambda_{j}$ denotes the tier dependent variable. The parameters for the respective tiers are given in Table 2. The spectrum efficiency of the categories of users described in Subsection 3.3 is given as: 


$$
\begin{aligned}
& \left\{\begin{aligned}
\log _{2}(1+ & \left.\frac{\mathrm{P}_{s, j, i} \Delta_{u, s, j, i}}{\sigma^{2}}\right), \forall j \rightarrow m \\
& \forall u_{s, i} \in\left\{\mathcal{E}_{s, m, i} \cup \mathcal{M}_{s, m, i}\right\} \\
& \forall i \in \mathcal{J}
\end{aligned}\right. \\
& \log _{2}\left(1+\frac{\mathrm{P}_{s, j, i} \Delta_{u, s, j, i}}{\sigma^{2}+\sum_{\substack{k \in\{Z\} \\
j \neq k}} \mathrm{P}_{s, k, i} \Delta_{u, s, k, i}}\right), \\
& \pi_{u, s, j, i}=\left\{\begin{array}{c}
j \neq k \\
Z=\mathcal{F}_{i}\left|j \rightarrow f_{i} ; \quad Z=\mathcal{P}_{i}\right| j \rightarrow P_{i}
\end{array}\right. \\
& \log _{2}\left(1+\frac{\mathrm{P}_{s, j \in c, i} \Delta_{u, s, j \in c, i}}{\sigma^{2}+\sum_{\substack{p^{\prime} \in c_{i}^{\prime} \\
c_{i} \neq c_{i}^{\prime}}} \sum_{\substack{k \in\left\{\mathcal{P F}_{i}^{\prime}\right\} \\
j \neq k}} \mathrm{P}_{s, k, i} \Delta_{u, s, k, i}}\right), \\
& \forall j \rightarrow p f, \quad \forall i \in \mathcal{J}, \\
& \forall u_{s, i} \in\left\{\mathcal{E}_{s, p f, i} \cup \mathcal{M}_{s, p f, i}\right\}
\end{aligned}
$$

where $\Delta_{u, s, j, i}$ denotes the channel gain of the downlink between an access point $j \in\{m, f, p, p f\}$ owned by InP $i$ and a user $u_{s, i}$ subscribed to SP $s \in \mathcal{S} ; \Delta_{u, s, j, i}$ is derived from (1). $\mathrm{P}_{s, j, i}$ indicates the downlink transmit power of the access point $j \in\{m, f, p, p f\}$ belonging to an $\operatorname{InP} i$; and $\sigma^{2}$ is the background noise. The double summation in (2) when $j \rightarrow p f$ indicates the associated intra-cluster interference, where a cluster $c$ comprises femtocells in the coverage of a picocell, and as such, the set of clusters is such that $\mathcal{C}_{i}=\left\{c_{i}\left|c_{i} \in \mathcal{N}, 1 \leq c_{i} \leq\right| \mathcal{P}_{i} \mid\right\}$.

\subsubsection{Highly mobile slice users}

As stated in Subsection 3.4, users engaged in V2X communications are modelled as URLLC slice users. The channel modelling components of highly mobile users comprise large-scale fading and small-scale fading components. The small-scale fading component is a result of the high mobility characterisation of the vehicles. For a slice user $u_{s, m, i}$ in the V2N (or V2I) communication layer, the large-scale propagation model is given as follows $[63,64]$ :

$$
\lambda_{u, s, j, i}=128.1+37.6 \log \left(\mathrm{d}_{u, s, j, i}\right), j \rightarrow m, i \in \mathcal{R}_{s, m, i}
$$

Herein, $\mathrm{d}_{u, s, j, i}$, denotes the distance between a slice user $u_{s, i}$ (i.e., a vehicle) and the macro-base-station $m_{i}$. Following categorisation of slice users in the V2X tier in Subsection 3.4, the large-scale fading propagation model of

Table 2 - Tier dependent variable for propagation model.

\begin{tabular}{|l|l|}
\hline Tier & $\Lambda_{j}(\mathrm{~dB})$ \\
\hline \hline$j \rightarrow m$ & 30 \\
\hline$j \rightarrow p$ & 35 \\
\hline$j \rightarrow f$ & 40 \\
\hline$j \rightarrow p f$ & 40 \\
\hline
\end{tabular}

slice users (i.e., vehicle) in the V2V-communication layer is given as [65]:

$$
\lambda_{u, w, m, i}=\left\{\begin{array}{c}
41+22.7 \log (3)+20 \log (h / 5), \\
\mathrm{d}_{u, s, w} \leq 3 \\
41+22.7 \log \left(\mathrm{d}_{u, s, w}\right)+20 \log (h / 5), \\
\mathrm{d}_{u, s, w} \leq \mathrm{d}_{\text {thres }} \\
9.45+40 \log \left(\mathrm{d}_{u, s, w}\right)+2.7 \log (h / 5) \\
-17.3 \log \left(\beta_{w}-1\right)\left(\beta_{u, s, m, i}-1\right), \\
\mathrm{d}_{\text {thres }} \leq \mathrm{d}_{u, s, w}
\end{array}\right.
$$

where $\mathrm{d}_{u, s, w}$ denotes the distance between a vehicle $u_{w} \in$ $\mathcal{W}$ engaged in transmission mode and another slice user (i.e., vehicle) $u_{s, m, i} \in \mathcal{R}_{s, m, i}$. The notations $h, \beta_{u, s, m, i}$, and $\beta_{w}$ denote the carrier frequency in $\mathrm{GHz}$; the effective antenna height of the vehicles in V2N and V2V communication layers. The distance threshold denoted as $\mathrm{d}_{\text {thres }}$ in (4) takes the following form [65]:

$$
\mathrm{d}_{\text {thres }}=\frac{4\left(\beta_{w}\right)\left(\beta_{u, s, m, i}\right) h / 5}{l}
$$

where $l$ represents the speed of light. Based on (4), the spectrum efficiency of URLLC users in the V2N communication layer is given as:

$$
\pi_{u, s, m, i}=\log _{2}\left(1+\frac{\mathrm{P}_{s, m, i} \Delta_{u, s p, m, i}\left|\zeta_{u, s, m, i}\right|^{2}}{\sigma^{2}+\sum_{\substack{w \in\{\mathcal{W}\} \\ w \neq u}} \mathrm{P}_{u, w} \Delta_{u, s, w}\left|\zeta_{u, w}\right|^{2}}\right)
$$

The small-scale fading components are captured in (6) by the notations $\zeta_{u, s, m, i}$ and $\zeta_{u, w}$. Note that the small-scale fast fading component is independent and identically distributed (i.i.d) as $\mathcal{C} \mathcal{N}(0,1)$. In Fig. 3, we give an illustration of the V2X tier.

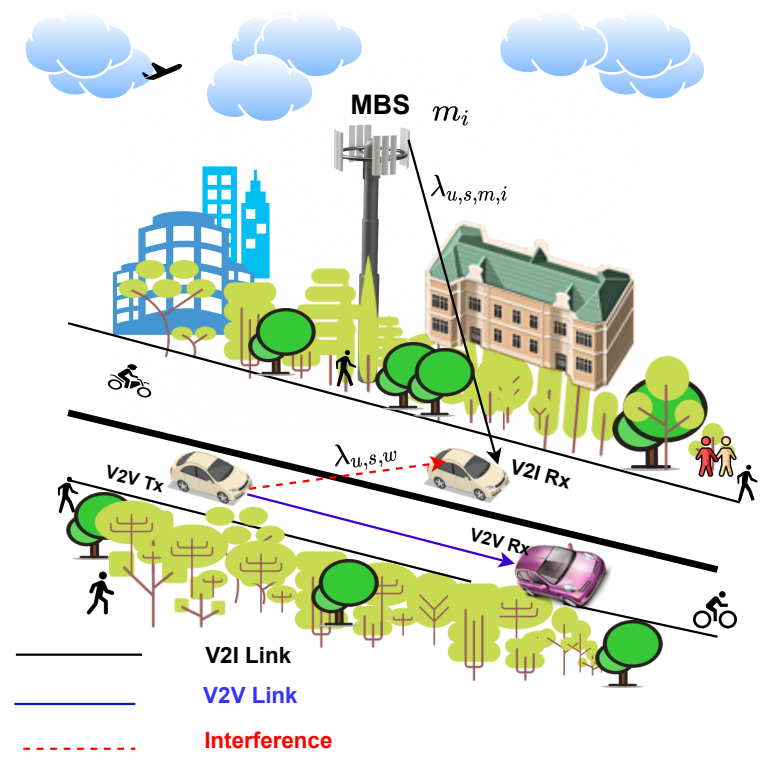

Fig. 3 - Illustration of the V2X-tier. 


\section{MULTI-DOMAIN GAMES FRAMEWORK}

Recently, mathematical economics and business management approaches have been employed in designing radio resource management frameworks $[7,66]$. To this end, the concepts of FM have played a significant role in the modelling of our M-TTSD network. Without loss of generality, we model an InP $i \in \mathcal{J}$ as a seller, an MVNO $v \in \mathcal{V}$, and an SP $s \in \mathcal{S}$ being buyer/seller depending on the hierarchical level in the M-TTSD network. This is illustrated in Fig. 4. Following the thesis of FM, we assume, the resources traded in the market are non-storable and divisible. For instance, spectrum or bandwidth can be traded in an M-TTSD network.

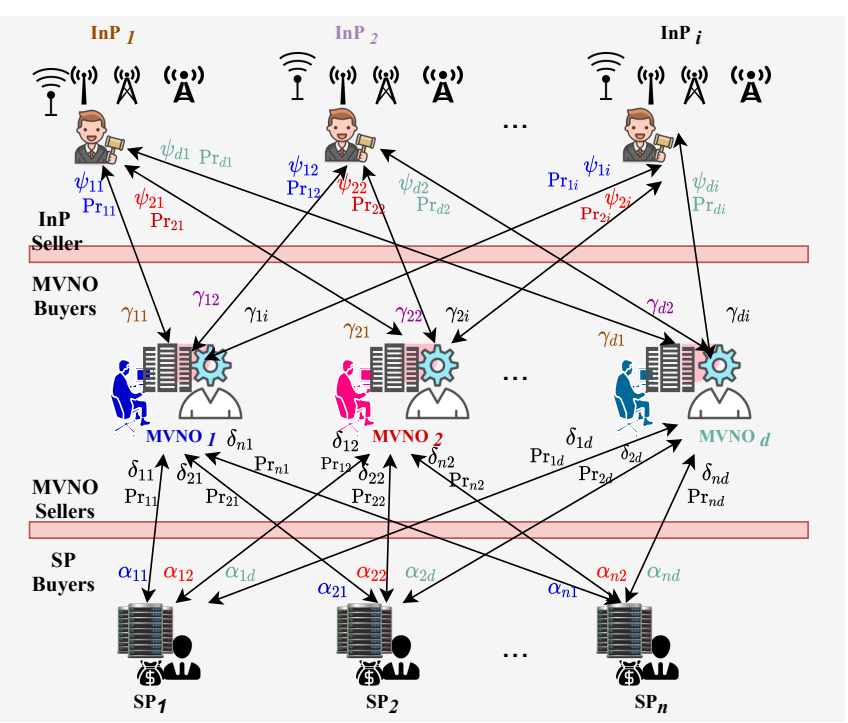

Fig. 4 - An illustration of the multi-domain games framework.

To design an efficient framework, we adopt a multistage auction mechanism owing to its ability to model interactions of the respective network entities as they trade network resources $[7,67]$. A simplistic information flow in Fig. 4 is shown in Fig. 5, which also may be adapted for a multiple resource scenario.

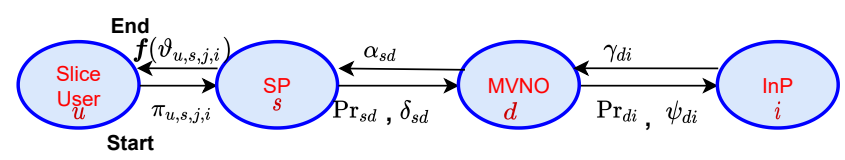

Fig. 5 - A simplistic illustration of information flow in the multi-domain games framework.

We consider a business model in which the InP $k \in \mathcal{J}$ has a set of resources such as radio spectrum, computational memory, given as $\mathcal{H}_{k}=\left(h_{1}, h_{2}, \ldots, h_{o}\right)$. Furthermore, the MVNOs bid for resources of the respective InPs $\mathcal{J}$, such that resources purchased by the MVNO set $\mathcal{V}$ is given as $\gamma=\left(\gamma_{1}, \gamma_{2}, \ldots, \gamma_{v}\right)$. We succinctly state that $\gamma_{\boldsymbol{d}}=\left(\gamma_{d, 1}, \gamma_{d, 2}, \ldots, \gamma_{d, i}\right)$ such that $d \in \mathcal{V}$ and $i \in \mathcal{J}$, additionally, $\gamma_{d, k}$ denotes the share of the resources of InP $k$ allocated to MVNO $d$. Given that an InP can have more than one resource type as explained earlier, thus $\gamma_{d, k}=$ $\left(H_{d, k, 1}, H_{d, k, 2}, \ldots, H_{d, k, o}\right)$, where $H_{d, k, o}$ is the share of re- source type $h_{o}$ owned by InP $k$ that is allocated to MVNO $d$ and $H_{d, k, o} \geq 0$. We assume, an InP and an MVNO can exercise their Individual Rationality (IR) when $H_{d, k, o}=0$. In an M-TTSD network, since an InP can trade its resources with interested MVNOs, hence

$$
\sum_{d \in \mathcal{V}} H_{d, k, o} \leq 1, \quad \forall k \in \mathcal{J}, \quad \forall h_{o} \in \mathcal{H}_{k} .
$$

An MVNO bids $\psi_{d, k}$ for the resources of an InP taking into consideration its resource preference factor $\operatorname{Pr}_{d, k, h_{o}}$. The resource preference $\operatorname{Pr}_{d, k, h_{o}}$ shows the importance of a particular resource $h_{o}$ owned by $\operatorname{InP} k$ to MVNO $d$. It is of the form given by:

$$
\sum_{h_{o} \in \mathcal{H}_{k}} \operatorname{Pr}_{d, k, h_{o}}=1, \quad \forall k \in \mathcal{J}, \forall d \in \mathcal{V}
$$

where $\operatorname{Pr}_{d, k, h_{o}} \geq 0$ and with $\operatorname{Pr}_{d, k, h_{o}}=0$ indicating that an MVNO is not interested in a particular resource of an InP. Moreover, $\psi_{d, k} \geq 0$ and if an MVNO bids $\psi_{d, k}=0$, this indicates the MVNO is not interested in bidding for a particular resource. To this end, the price of a resource $\rho_{h_{o}, k}$ of an InP $k$ is expressed as:

$$
\rho_{h_{o}, k}=\sum_{d \in \mathcal{V}} \psi_{d, k} \operatorname{Pr}_{d, k, h_{o}}, \quad \forall k \in \mathcal{J}, \quad h_{o} \in \mathcal{H}_{k}
$$

Thus, the share of resource $h_{o}$ of InP $k$ allocated to MVNO $d$ is given as:

$$
H_{d, k, o}=\frac{\psi_{d, k}}{\rho_{o, k}} \operatorname{Pr}_{d, k, h_{o}}
$$

Next, we take a close-up view of the bidding vector of a MVNO. To this end, an MVNO $d$ can bid for resources from different InPs such that for ease of exposition, the bidding vector $\boldsymbol{\psi}_{\boldsymbol{d}}=\left(\psi_{d, 1}, \psi_{d, 2}, \ldots, \psi_{d, i}\right)$. On the other hand, if we denote the bidding budget of an MVNO by $\theta_{d}$, hence

$$
\sum_{k \in \mathcal{J}} \psi_{d, k} \leq \theta_{d}, \quad \forall d \in \mathcal{V}
$$

In this case, $\psi_{d, k}$ is such that it creates IC for an InP $k$, otherwise, resource trading cannot take place. We also consider the MVNO-SP auction stage. In this case, a set of SPs bids for the resources of an MVNO. Thus, $\alpha=\left(\boldsymbol{\alpha}_{1}, \boldsymbol{\alpha}_{2}, \ldots, \boldsymbol{\alpha}_{\boldsymbol{s}}\right)$ such that $\boldsymbol{\alpha}_{\boldsymbol{n}}=\left(\alpha_{n, 1}, \alpha_{n, 2}, \ldots, \alpha_{n, e}\right)$, with $\alpha_{n, e}$ denoting the share of the resources MVNO $e \in$ $\mathcal{V}$ allocated to SP $n \in \mathcal{S}$. Given that an MVNO can have more than one resource type, therefore, $\alpha_{n, e}=$ $\left(L_{n, e, 1}, L_{n, e, 2}, \ldots, L_{n, e, l}\right)$. Here, $L_{n e l}$ is the share of resource $L_{l}$ allocated to SP $n \in \mathcal{S}$ by MVNO $e \in \mathcal{V}$. Hence, in the M-TTSD network, the resources allocated by an MVNO to a set of SPs is such that:

$$
\sum_{n \in \mathcal{S}} L_{n, e, l} \leq 1, \quad \forall e \in \mathcal{V}, \forall l \in \mathcal{L}
$$


Next, we consider the preference of SP $n \in \mathcal{S}$ for the resources of MVNO $e \in \mathcal{V}$. If we denote preference factor by $\operatorname{Pr}_{n, e, l}$, then

$$
\sum_{l \in \mathcal{L}} \operatorname{Pr}_{n, e, l} \leq 1, \quad \forall e \in \mathcal{V}, \forall n \in \mathcal{S},
$$

The bid $\delta_{n, e}$ placed by an SP $n \in \mathcal{S}$ to an MVNO $e \in \mathcal{V}$ is such that $\delta_{n, e} \geq 0$. With $\operatorname{Pr}_{n, e, l}=0$, then SP $n \in \mathcal{S}$ does not place a bid for MVNO $e \in \mathcal{V}$ resources. Besides, $\delta_{\text {ne }}$ is such that it is IC for MVNO $e \in \mathcal{V}$, otherwise, the bid is rejected. Hence, resource price of the MVNO $e \in \mathcal{V}$ is such that:

$$
\phi_{e, l}=\sum_{n \in \mathcal{S}} \delta_{n, e} \operatorname{Pr}_{n, e, l}, \quad e \in \mathcal{V}, l \in \mathcal{L}
$$

Thus, the share of resource type $L_{n, e, l}$ an SP $n \in \mathcal{S}$ receives from an MVNO $e \in \mathcal{V}$ is given as:

$$
L_{n, e, l}=\frac{\delta_{n, e}}{\phi_{e, l}} \operatorname{Pr}_{n, e, l}
$$

If we denote the bidding vector of an SP $n \in \mathcal{S}$ as $\boldsymbol{\delta}_{\boldsymbol{n}}=$ $\left(\delta_{n, 1}, \delta_{n, 2}, \ldots, \delta_{n, e}\right)$ owing to the ability of an SP to bid for resources from several MVNOs, thus:

$$
\sum_{e \in \mathcal{V}} \delta_{n, e} \leq \mu_{n}, \forall n \in \mathcal{S P}
$$

where $\mu_{n}$ denotes the bidding budget of an SP. This implies that the utility of the MVNO is based on the revenues accrued from the auction of network resources to SPs. On the other hand, SPs provide different slice use-cases contracts to subscribed users. It is important to reiterate that InPs, MVNOs, and SPs are individually rational and consider the IC of a bid offered before they release their resources.

For ease of exposition, an MVNO $e \in \mathcal{V}$ in the InP-MVNO hierarchical stage maximises its utility $\mathcal{U}_{e}$ derived from its bidding vector $\psi_{e}$ in the form given as:

$$
\mathcal{u}_{e}=\arg \max \left(\frac{\psi_{e, 1}}{\psi_{e, 1}+\psi_{e, 1}^{\prime}}, \frac{\psi_{e, 2}}{\psi_{e, 2}+\psi_{e, 2}^{\prime}}, \cdots, \frac{\psi_{e, i}}{\psi_{e, i}+\psi_{e, i}^{\prime}}\right)
$$

$$
\begin{array}{ll}
\text { s.t. } & \sum_{i \in \mathcal{J}} \psi_{e, i} \leq \theta_{e}, \quad \forall e \in \mathcal{V} \\
\text { C2: } \quad \sum_{k \in \mathcal{J}} \mathcal{B}_{k} \gamma_{e, k} \geq \sum_{q \in \mathcal{S}} \chi_{q}, \quad \forall e \in \mathcal{V} \\
\text { C3: } \quad \psi_{e, i} \geq 0, \quad \forall e \in \mathcal{V}, \quad \forall i \in \mathcal{J}
\end{array}
$$

where the sum of the bids of other MVNOs $e \neq e^{\prime}$ is denoted by $\psi_{e, i}^{\prime}$. Constraint $\mathrm{C} 1$ ensures the sum of the bids of an MVNO for the resources of the respective InPs does not exceed its budget $\theta_{e}$. Constraint C 2 ensures that the total load demand for a set of SPs bidding for the resources of the MVNO is met by the sum of resources purchased by the MVNO from the respective InPs. Constraint C3 ensures that a bid value is non-negative and also an MVNO can decide not to place a bid such that $\psi_{e, i}=0$.
Similarly, at the SP-MVNO hierarchical layer, the utility $\mathcal{U}_{n}$ of SP $n \in \mathcal{S}$ playing the multi-domain multi-tenant game is of the form given as:

$$
\mathcal{U}_{n}=\arg \max \left(\frac{\delta_{n, 1}}{\delta_{n, 1}+\delta_{n, 1}^{\prime}}, \frac{\delta_{n, 2}}{\delta_{n, 2}+\delta_{n, 2}^{\prime}}, \cdots, \frac{\delta_{n, e}}{\delta_{n e}+\delta_{n, e}^{\prime}}\right)
$$

$$
\begin{array}{ll}
\text { S4.t. } & \sum_{e \in \mathcal{V}} \delta_{n, e} \leq \mu_{n} \quad \forall n \in \mathcal{S} \\
\text { C5: } & \sum_{n \in \mathcal{S}} \mathcal{C}_{e} \alpha_{n, e} \geq \sum_{u \in \mathcal{S}_{s p}} v_{u} \quad \forall e \in \mathcal{V} \\
\text { C6: } & \delta_{n, e} \geq 0 \quad \forall n \in \mathcal{S}, \quad \forall e \in \mathcal{V}
\end{array}
$$

where the sum of bids of other SPs $n \neq n^{\prime}$ is denoted by $\delta_{n}^{\prime}, e$. We guarantee that the bid budget of an SP is not exceeded in Constraint C4. In constraint C5, the demands of the respective subscribers are met by their SP. Lastly, in constraint $\mathrm{C} 6$, a bid value is ensured to be non-negative and also an SP can decide not to place a bid such that $\delta_{n, e}=0$.

\section{RESOURCE ALLOCATION FRAMEWORK}

In this section, we describe the service provider-slice users' communication layer, the latency and delay model employed in this work and the resource allocation framework.

\subsection{Service Provider-slice user layer}

In this work, without loss of generality, it is assumed that a slice user $u_{s, i} \in\{\mathcal{E} \cup \mathcal{M} \cup \mathcal{R}\}$ is subscribed to one SP $g \in \mathcal{S}$ at a time. Moreover, this assumption is downplayed by the ability of SPs to bid for resources from different MVNOs, and by extension InPs as the case may be. To this end, the QoS requirements of the respective slice users are satisfactorily met. Furthermore, the QoS requirements of the slice users are dynamically met by taking into consideration the cell load, tier load, bid budget, associated interference, slice users' distribution and location, slice usecase QoS requirement, delay and latency thresholds.

\subsection{Latency and delay model}

In a similar trend with the work in [68], the link layer model and effective capacity theory in the seminal work of $[60,61]$ are employed in addressing latency and service rate requirements of slice users. The link layer model $[60,61]$ gained wide acceptance due to the ease of implementation, and uncomplicated translation of decisive QoS requirements such as delay bounds, probability of packet loss, and packet arrival rate. While the effective capacity of a slice ensures that the QoS requirements are met at a maximum packet arrival rate, it leverages the average packet $\eta_{s, h, i}$, maximum delay bound threshold $D_{\text {max }}$, a delay-bound violation probability $\xi$. Thus, the effective capacity for a slice user $u_{s, j, i} \in\{\mathcal{M} \cup \mathcal{R}\}$ is given as $[68,61]$ : 


$$
\chi\left(v_{u, s, i}\right)=\lim _{t \rightarrow \infty} \frac{1}{t} \frac{1}{v_{u, s, i}} \log \mathbb{E}\left[e^{v_{u, s, i} Q_{u, s, i}^{t}}\right]
$$

where the QoS exponent is denoted by $v_{u, s, i}$, and the source packet arrival rate over the time interval $[0, t)$ is denoted by $Q_{u, s, i}^{t}$. By employing the theory of large deviations [69] and the application of a moment generating function [70] to (19), the work in $[68,61]$, shows that the minimum achievable rate $\vartheta_{u, s, i}^{\text {thres }}$ of a slice $u_{s, j, i} \in\{\mathcal{M} \cup \mathcal{R}\}$ can be expressed as:

$$
\vartheta_{u, s, i}^{\text {thres }}=-\frac{L_{u, s p, i} \log (\xi)}{D_{\max } \log _{e}\left(1-\frac{\log (\xi)}{D_{\max } \eta_{u, s, i}}\right)}
$$

where the packet size and delay bound violation probability threshold are denoted as $L_{u, s, i}$ and $\xi$, respectively.

\subsection{Resource allocation model}

Herein, to meet slice users' demands, the resources from the respective MVNOs and by extension, the InPs, are pooled by an SP and then allocated to slice users in a dynamic manner similar to the work in [68]. However, unlike [68], we consider a distributed resource allocation framework in which an SP has control over its allocation process rather than a centralised approach. Moreover, the utility of a slice user $\boldsymbol{f}\left(\vartheta_{u, s, j, i}\right)$ is expressed as $[71,20,67]$ :

$$
\boldsymbol{f}\left(\vartheta_{u, s, j, i}\right)=\left\{\begin{array}{l}
\frac{\vartheta_{u, s, j, i}^{1-b}}{1-b}, \quad b \neq 1 \\
\log \left(\vartheta_{u, s, j, i}\right), \quad b=1
\end{array}\right.
$$

where $\boldsymbol{f}\left(\vartheta_{u, s, j, i}\right)$ is expressed as:

$$
\vartheta_{u, s, j, i}=\mathcal{Y}_{i, s} \cdot T_{s, j, i} \cdot \varphi_{u, s, j, i} \cdot \pi_{u, s, j, i}
$$

The weight of a resource type belonging to InP $i$ allocated to SP $s$ is denoted by $\mathcal{Y}_{i, s}$. We represent the tier-slice ratio of the resource by $T_{s, j, i}$, and $\varphi_{u, s, j, i}$ denotes the slice user ratio. The spectrum efficiency $\pi_{u, s, j, i}$ is given in (2) and the spectrum efficiency $\pi_{u, s, j, i}$ is strictly concave and strictly increases i.e., non-decreasing values of $b \geq 0$. In this work, we adopt logarithmic utility in (22) when $b=1$. The logarithmic utility ensures proportional fair rate allocations.

\section{PROBLEM FORMULATION}

In this section, a latency-aware, bid-aware, and distributed resource allocation problem in the hierarchical layers of an M-TTSD network is examined. First, the overall utility of an SP is investigated by formulating a utility maximisation problem in the SP-user hierarchical layer. We adopt additive utility given in [72, 73] to determine the overall utility of an SP. Additive utility assumes preferential independence between attributes such that the total associated utility of an SP is the aggregation of the utility of individual slice users.
In order to fully maximise the utility of the respective SP in the M-TTSD network, we formulate a joint tier-domain user-association SP-slice user resource allocation problem in the (P1).

Constraint (23)a guarantees that the minimum achievable rate for each slice use-case is met for all the slice users described in the categorisation in Subsection 3.3 and for the respective tiers and domains. Constraint (23)b ensures that a slice user is associated with only one access network in a tier and by extension, can only be associated with one domain network. Constraint (23)c ensures the domain-slice user association restriction such that, a user $u$ subscribed to SP $s$ is associated with domain $i$ when $\Upsilon_{i, s, u}=1$, and otherwise not associated with the domain with $Y_{i, s, u}=0$. A user can only be associated with one domain only. Similarly, Constraint (23)d enforces the tier-slice user association restriction. A slice user $u$ subscribed to SP $s$ is associated with a tier $j$ when $\Omega_{j, s, u}=1$, and otherwise when $\Omega_{j, s, u}=0$. Moreover, constraints (23)e - (23)h highlight that the sum of slice user ratio $\varphi_{u, s, j, i}$ of a resource does not exceed the resource size for respective tiers. Besides, constraints (23)i - (23)l indicate the slice user ratio which is the fractional allocation of resources allocated to a slice user. To this end, the slice user ratio lies between 0 and 1 , hence, it is a positive value. Furthermore, we investigate the resource allocation challenge in the MVNO hierarchical layer. We formulate a utility maximisation optimisation problem in MVNO-SP layer by employing additive utility similar to the SP-slice user utility maximisation problem in (P2). The total utility of MVNO $d$ is the aggregation of the utility of SPs related to the resources of MVNO $d$. Constraint (24)a guarantees that aggregation of resource $\alpha_{n, k}$ allocated to SP $n$ by MVNO $d$ does not exceed the resource $\gamma_{d k}$ bid for by MVNO $d$ from InP $k$. Besides, constraint (24)b establishes the Business-to-Business (B2B) relationship $\nabla_{n, k}$ between an SP $n$ and an InP $k$. When a slice user subscribed to SP is associated with a domain network managed by an InP $k, \nabla_{n, k}=0$, otherwise resources of InP $k$ cannot be utilised. In addition, constraint (24)c ensures the resource share $\alpha_{n, k}$ of MVNO $k$ resource allocated to SP $n$ lies between 0 and 1. Moreover, the IC for MVNOs is guaranteed in constraint (24)d. To this end, the sum bids $\delta_{n, d}$ of SPs associated with MVNO $d$ must exceed the sum of the bids $\psi_{d k}$ placed by MVNO $d$ for the resources of InPs associated with it. 


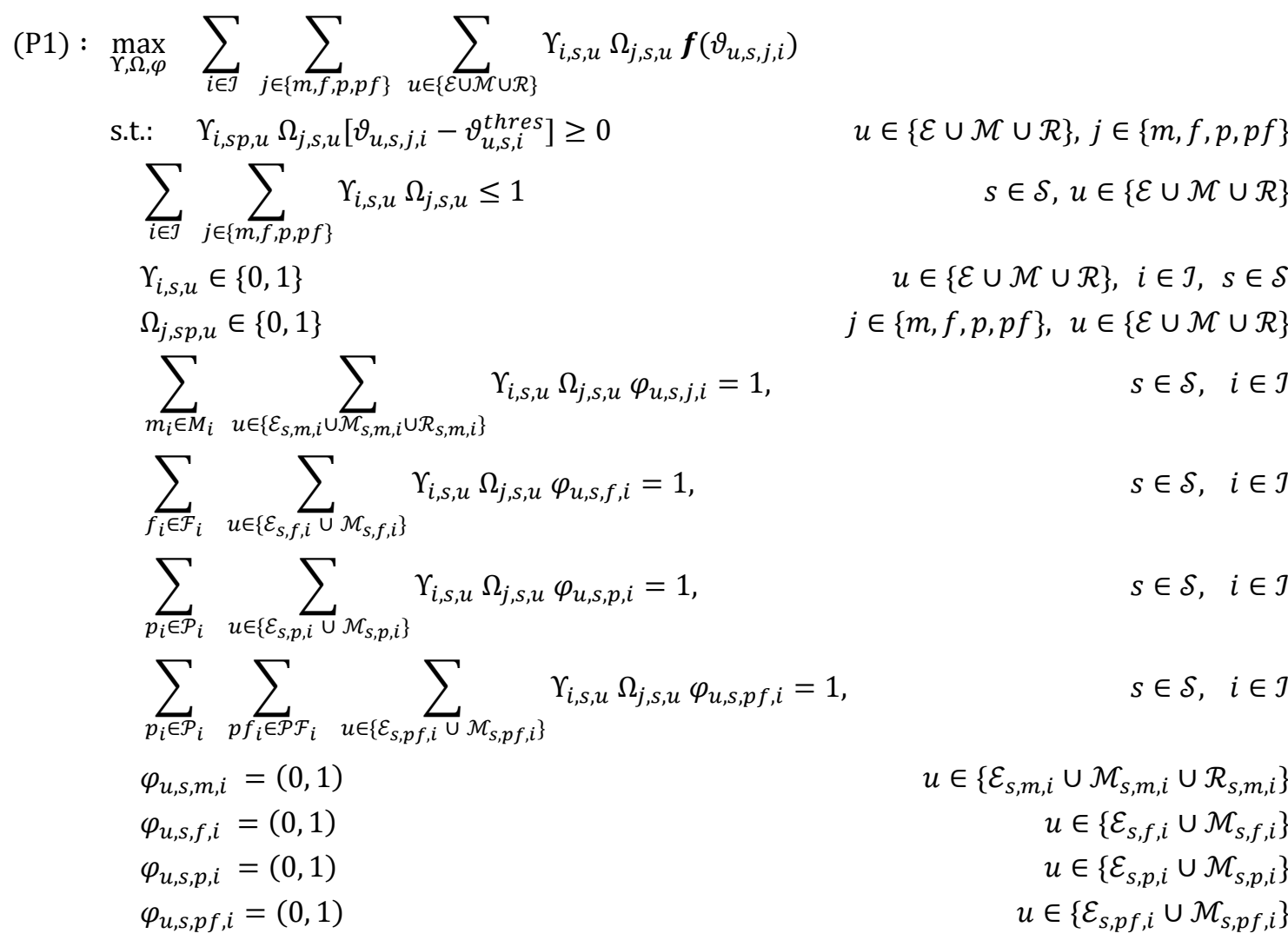

(P2) : $\max _{\Upsilon, \Omega} \quad \sum_{n \in \mathcal{S}} \delta_{n, d}\left(\sum_{k \in \mathcal{J}} \nabla_{n, k} \alpha_{n d} \gamma_{d k} \mathcal{B}_{k}\right)$

$$
\begin{aligned}
& \text { s.t.: } \sum_{n \in \mathcal{S} \mathcal{P}} \alpha_{n d} \leq \gamma_{d k} \\
& \nabla_{n, k} \in\{0,1\} \\
& \alpha_{n, k} \in(0,1) \\
& \sum_{n \in \mathcal{S}} \delta_{n, d} \geq \sum_{k \in \mathcal{J}} \psi_{d k}
\end{aligned}
$$

\section{PROPOSED SOLUTIONS}

In this section, we give an in-depth description of the proposed solutions to the latency and bid aware dynamic resource allocation problem in an M-TTSD network stated in (P1) and (P2). First, the objective function in (P1) is reformulated for tractability and complexity reduction using the hierarchical decomposition technique [74]. For clarity, readability, and without the abuse of notations, in the reformulation of (P1), we drop indexes in $Y_{i, s, u}, \Omega_{j, s, u}$ and $\boldsymbol{f}\left(\vartheta_{u, s, j, i}\right)$. (P1) is transformed into (P1') taking into consideration the physical network described in Subsection 3.2 and the categories of slice users in Subsection 3.3.

$$
\begin{array}{r}
\left(\mathrm{P}^{\prime}\right): \max _{\Upsilon, \Omega} \sum_{i \in \mathcal{J}} \sum_{m \in M} \sum_{u \in\{\mathcal{E} \cup \mathcal{M} \cup \mathcal{R}\}} \Upsilon_{i, u} \Omega_{m, u} \boldsymbol{f}\left(\vartheta_{u, m, i}\right) \\
+\sum_{i \in \mathcal{J}} \sum_{f_{i} \in \mathcal{F}_{i}} \sum_{u \in\{\mathcal{E} \cup \mathcal{M}\}} \Upsilon_{i, u} \Omega_{f, u} \boldsymbol{f}\left(\vartheta_{u, f, i}\right) \\
+\sum_{i \in \mathcal{J}} \sum_{p_{i} \in \mathcal{P}_{i}} \sum_{u \in\{\mathcal{E} \cup \mathcal{M}\}} \Upsilon_{i, u} \Omega_{p, u} \boldsymbol{f}\left(\vartheta_{u, p, i}\right) \\
+\sum_{i \in \mathcal{J}} \sum_{p_{i} \in \mathcal{P}_{i}} \sum_{p f_{i} \in \mathcal{P}_{\mathcal{F}_{i}}} \sum_{u \in\{\mathcal{E} \cup \mathcal{M}\}} \Upsilon_{i, u} \Omega_{p f, u} \boldsymbol{f}\left(\vartheta_{u, p f, i}\right)
\end{array}
$$


For ease of exposition, we give an in-depth breakdown of ( $\left.\mathrm{P} 1^{\prime}\right)$ by employing hierarchical decomposition [74] in Appendix A. We proceed by addressing the slice user multi-tier multi-domain association problem ingrained in $\left(\mathrm{P} 1^{\prime}\right)$.

\subsection{Slice user multi-tier multi-domain associ- ation problem}

Furthermore, we address the slice user multi-tier multidomain association problem in an M-TTSD network by adopting the following approaches: (i) QoS in multidomain networks [75], and (ii) association of users in heterogeneous networks [76, 77]. To this end, we formulate the association problem in an M-TTSD network as a maximisation optimisation problem given in subproblem (P3) as:

$$
\begin{aligned}
\text { (P3) : } \max _{\Upsilon, \Omega} \sum_{i \in \mathcal{J}} \sum_{j \in\{m, f, p, p f\}} \sum_{u \in\{\varepsilon \cup \mathcal{M} \cup \mathcal{R}\}} \Upsilon_{i, s, u} \Omega_{j, s, u} \\
\text { s.t.: } \sum_{i \in \mathcal{J}} \sum_{j \in\{m, f, p, p f\}} \Upsilon_{i, s, u} \Omega_{j, s, u} \leq 1, \forall u, \forall s \\
\Upsilon_{i, s, u} \in\{0,1\} \quad \forall i, \forall u, \forall s \\
\Omega_{j, s, u} \in\{0,1\} \quad \forall j, \forall u, \forall s
\end{aligned}
$$

In addressing sub-problem (P3), we consider the peculiarities of each tier such as slice types it can accommodate, coverage radius, transmit power. We ensure that a slice user can only associate with one tier and by extension associate with only one domain network by ensuring constraint (25)a is enforced. Moreover, constraints (25)b and (25)c help to relax constraint (25)a by ensuring the sum of the products in (25)a equals one for a slice user to be admitted to a tier in a particular domain network. It is important to note that the expressions (50)(53) were employed in the breakdown of the objective function in sub-problem (P3). Additionally, the manyto-one concept in matching theory $[78,79]$ is adopted in our proposed multi-tier multi-domain slice user algorithm. This is a result of the practicability in M-TTSD networks. Fig. 6 illustrates the multi-tier multi-domain slice user matching algorithm proposed to solve subproblem (P3). Moreover, we give the pseudo-code of the multi-tier multi-domain slice user algorithm in Alg.1. For ease of exposition, expression (26) gives an insight into the mathematical representation of matching matrix for a slice $u \in\left\{\mathcal{E}_{s, p f_{i}, i} \cup \mathcal{M}_{s, p f_{i}, i}\right\}$. In Alg.1, $\boldsymbol{\Theta}=$ $\left\{\pi_{u, s, m, i}, \pi_{u, s, p, i}, \pi_{u, s, p f, i}, \pi_{u, s, m, k}, \pi_{u, s, p, k}, \pi_{u, s, p f, k}\right\}$.

Additionally, $\boldsymbol{\Pi}=\left\{\pi_{u, s, m, i}, \pi_{u, s, f, i}, \pi_{u, s, m, k}, \pi_{u, s, f, k}\right\}, \boldsymbol{\Delta}=$ $\left\{\pi_{u, s, m, i}, \pi_{u, s, p, i}, \pi_{u, s, m, k}, \pi_{u, s, p, k}\right\}, \boldsymbol{\Lambda}=\left\{\pi_{u, s, m, i}, \pi_{u, s, m, k}\right\}$. For ease of exposition, expression (26) gives an insight into the mathematical representation of matching matrix for a slice $u \in\left\{\mathcal{E}_{s, p f_{i}, i} \cup \mathcal{M}_{s, p f_{i}, i}\right\}$.

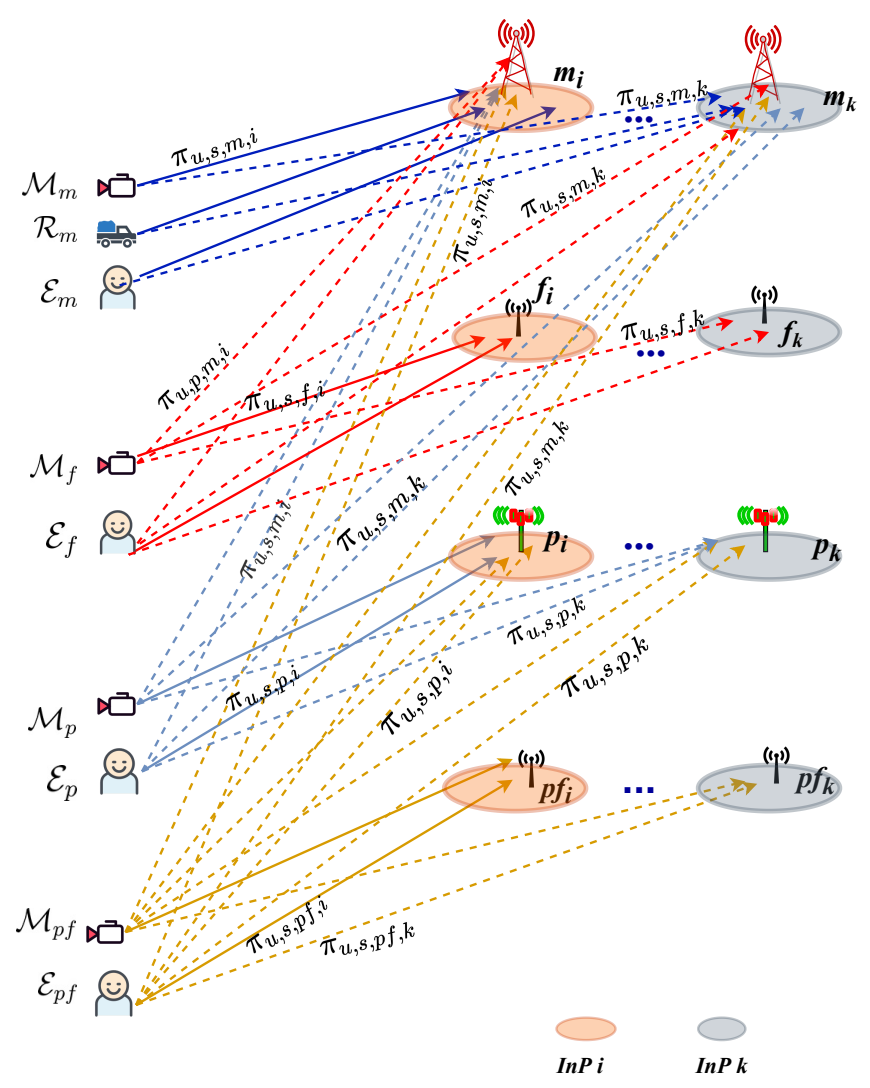

Fig. 6 - Multi-tier multi-domain slice user matching game.

$$
\mathbf{A}=\left[\begin{array}{ccc}
\Omega_{p f_{1}} & \Omega_{p_{1}} & \Omega_{m_{1}} \\
\Omega_{p f_{2}} & \Omega_{p_{2}} & \Omega_{m_{2}} \\
\vdots & \vdots & \vdots \\
\Omega_{p f_{i}} & \Omega_{p_{i}} & \Omega_{m_{i}}
\end{array}\right] \odot\left[\begin{array}{ccc}
\Upsilon_{1, p f_{1}} & \Upsilon_{1, p_{1}} & \Upsilon_{1, m_{1}} \\
\Upsilon_{2, p f_{2}} & \Upsilon_{2, p_{2}} & \Upsilon_{2, m_{2}} \\
\vdots & \vdots & \vdots \\
\Upsilon_{i, p f_{i}} & \Upsilon_{i, p_{i}} & \Upsilon_{i, m_{i}}
\end{array}\right]
$$

Solving the multi-tier multi-domain user association sub-problem (P3) gives room to further address the utility maximisation problem stated in $\left(\mathrm{P}^{\prime}\right)$. To this end, we further transform $\left(\mathrm{P}^{\prime}\right)$ to reflect variables of the utility function given in expressions (21)-(22) and user association carried out in solving sub-problem (P3). Therefore, we rewrite $\left(\mathrm{P}^{\prime}\right)$ to give $\left(\mathrm{P} 1^{\prime \prime}\right)$ in (27). It is important to note that logarithmic rule is applied to the expression (22) to reduce the complexity of solving ( $\left.\mathrm{P}^{\prime \prime}\right)$, thereby splitting the decision variables of $T_{s, j, i}$ and $\varphi_{u, s, j, i}$. 


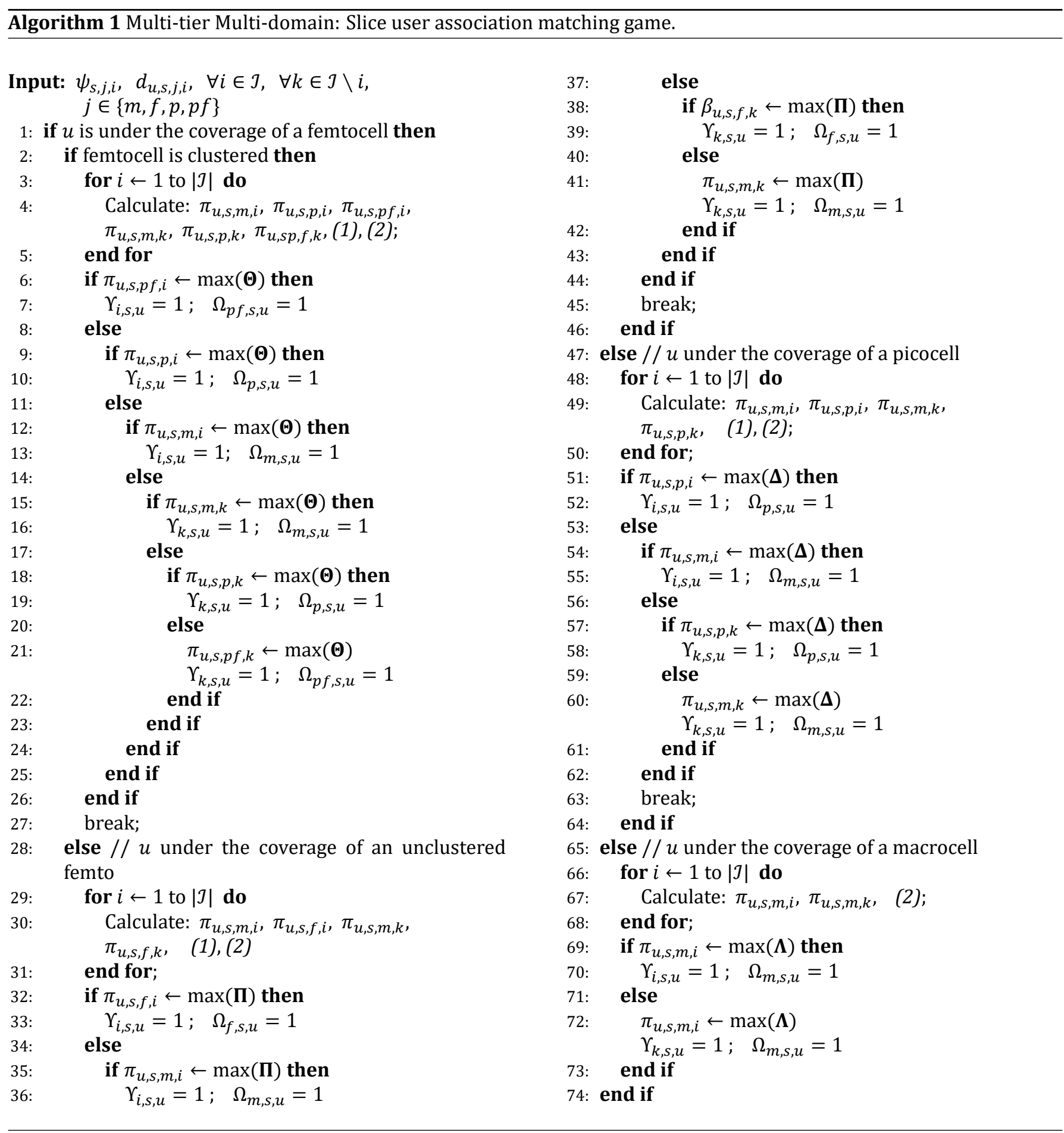




$$
\begin{aligned}
& \left(\mathrm{P} 1^{\prime \prime}\right): \max _{T_{s, j, i},} \varphi_{u, s, j, i} \\
& \sum_{i \in \mathcal{J}} \sum_{m \in M} \sum_{u \in\{\mathcal{E} \cup \mathcal{M} \cup \mathcal{R}\}}\left[\log \left(\mathcal{Y}_{i, s} T_{s, m, i} \pi_{u, s, m, i}\right)+\log \left(\varphi_{u, s, m, i}\right)\right]+\sum_{i \in \mathcal{J}} \sum_{f_{i} \in \mathcal{F}_{i}} \sum_{u \in\{\mathcal{E} \cup \mathcal{M}\}}\left[\log \left(\mathcal{Y}_{i, s} T_{s, f, i} \pi_{u, s, f, i}\right)+\log \left(\varphi_{u, s, f, i}\right)\right] \\
& +\sum_{i \in \mathcal{J}} \sum_{p_{i} \in \mathcal{P}_{i}} \sum_{u \in\{\mathcal{E} \cup \mathcal{M}\}}\left[\log \left(\mathcal{Y}_{i, s} T_{s, p, i} \pi_{u, s, p, i}\right)+\log \left(\varphi_{u, s, p, i}\right)\right]+\sum_{i \in \mathcal{J}} \sum_{p_{i} \in \mathcal{P}_{i}} \sum_{p}\left[\log \left(\mathcal{Y}_{i, s} T_{s, p, i} \pi_{u, s, p, i}\right)\right] \\
& +\sum_{i \in \mathcal{J}} \sum_{p_{i} \in \mathcal{P}_{i}} \sum_{\left.f_{i} \in \mathcal{E} \cup \mathcal{M}\right\}} \sum_{\left.\mathcal{F}_{i}\right\}}\left[\log \left(\varphi_{u, s, p, i}\right)\right]
\end{aligned}
$$

\subsection{SP-MVNO resource allocation}

It is important to note that $\mathcal{Y}_{i, s}$ is pivotal in slicing the resource in (27). Solving $\mathcal{Y}_{i, s}$ entails the SP-MVNO hierarchical layer described in Section 4. For a simple case of a single resource (i.e., bandwidth) and from Section $4, \mathcal{Y}_{i, s}$ is given as:

$$
\mathcal{Y}_{i, n}=\sum_{d \in \mathcal{H}} \mathcal{B}_{i} \gamma_{d, i} \alpha_{n, d}, \quad n \in \mathcal{S}, \quad i \in \mathcal{J}
$$

where $\mathcal{B}_{i}$ is the overall bandwidth size of $\operatorname{InP} i$. Moreover, the fraction of the resource allocated to MVNO $d$ by InP $i$ is represented as $\gamma_{d, i}$. Additionally, MVNO $d$ allocates a fraction $\alpha_{n, d}$ of its resources from InP $i$ to SP $n$. From (10) and (15), the values of $\gamma_{d, i}$ and $\alpha_{n, d}$ are dependent on the respective bids $\psi_{d, k}$ and $\delta_{n, d}$ placed by the MVNOs and the SP. Careful inspection and solution of (28) yields a solution to the resource allocation problem in the SPMVNO hierarchical layer, and by extension problem (P2) and its associated constraints given in (24)a-(24)d. To this end, we obtain optimal value of $\mathcal{Y}_{i, n}$ by adopting a recursive distributed backtracking technique $[80,81]$ for respective SPs. The recursive distributed backtracking relies on partial or incomplete information, which is a primary feature in the hierarchical layers of the M-TTSD network [82, 83]. Moreover, we employ the recursive backtracking technique owing to its ease of implementation, lightness of codes involved, and intuitiveness.

Additionally, it is generally employed to solve a constraint satisfaction problem [84]. However, we avoid the thrashing challenge peculiar with backtracking by ensuring that the bids by respective network players are IC, and also the right of players to display IR is guaranteed [84]. In Fig. 7, we illustrate the B2B relationships among SP, MVNO, and InPs in the resource allocation problem. In Alg. 2, we give the pseudo-codes of the recursive distributed backtracking algorithm.

Herein, $\epsilon_{s, k}$ in Alg. 2 represents the traffic load of slice users associated with a domain network $k$ but subscribed to the services of SP $s$. Moreover, $\omega_{s, d}$ denotes the preference of an SP $s$ for the resources of an MVNO $d$. In Lemma 1 , we give the characterisation of $\epsilon_{s, k}$ and by extension in Alg. 2.

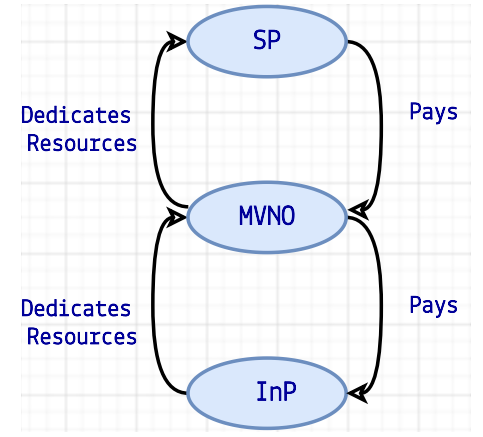

Fig. 7 - An illustration of the B2B model of a simple multiplayer network.

Lemma 1. We denote the average number of slice users at a specific time by $N$; $\tau$ as the mean call arrivals per unit time and we also denote the mean (palm) call duration by $Z$. If we assume the volume of data transmitted (i.e., in bits) during one call duration is $1 / \phi$, therefore, we can express the average traffic demand $\epsilon_{s, k}$ (bits/seconds) as:

$$
\epsilon=\frac{1}{\phi} \cdot \tau
$$

Therefore, the average throughput $\kappa$ (i.e. in bits/seconds) is given as:

$$
\kappa=\frac{\frac{1}{\phi}}{Z}
$$

By applying Little's Law [85] the number of slice user $N$ served at specific time can be expressed in the form:

$$
N=\tau \cdot Z
$$

We rewrite (31) by multiplying it by the mean data volume for the duration of a call $1 / \phi$. We have:

$$
\frac{1}{\phi} \cdot \frac{1}{Z}=\frac{\tau}{N} \cdot \frac{1}{\phi}
$$

By substituting (30) into (32), we have:

$$
\kappa=\frac{\tau}{\phi \cdot N}
$$

Besides, if we substitute (29) into (33) we have:

$$
\kappa=\frac{\epsilon}{N}=\frac{\text { mean traffic demand }}{\text { avg. no. of slice users served }} .
$$


Therefore, the average traffic demand $\epsilon$ is given as:

$$
\epsilon=\kappa \cdot N
$$

Algorithm 2 Distributed recursive backtracking for multi-player multi-domain game.

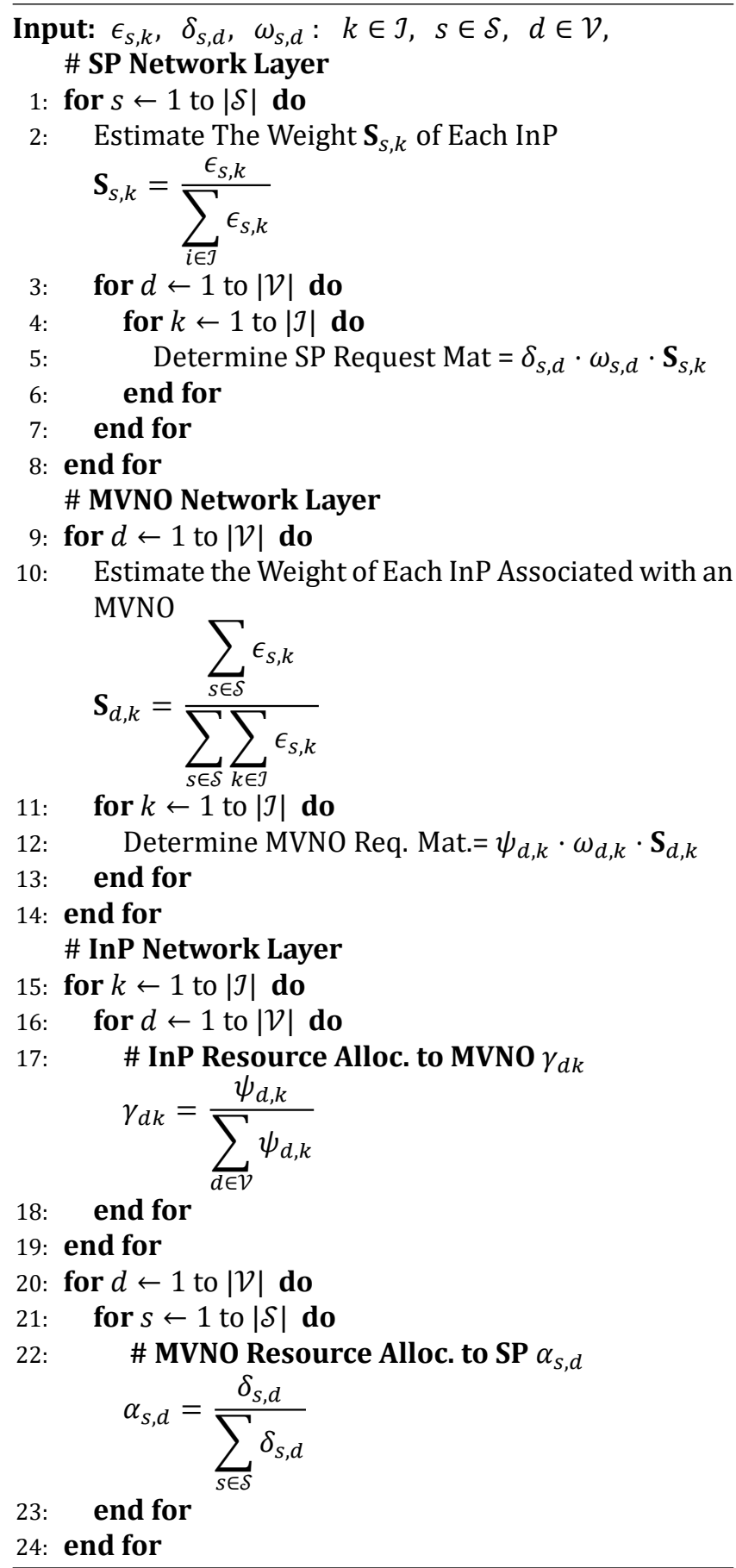

With the aid of Lemma 1, the average traffic demand of an M-TTSD network can be determined via Theorem 1.

Theorem 1. Let the mean user throughput given in (20) be the same as $\kappa$ in Lemma 1. Then, the mean traffic load of an SP $n \in \mathcal{S}$ wrt an InP $i \in \mathcal{J}$ is the sum of the mean traffic load of all the tiers and is of the form:

$$
\varrho_{n, i}=\sum_{j \in m, f, p f, p} \varrho_{n, j, i}, \quad \forall n \in \mathcal{S}, \quad i \in \mathcal{J}
$$

The mean traffic load of the macro-tier $\varrho_{n, m, i}$ is expressed as:

$$
\begin{aligned}
& \varrho_{n, m, i}= \sum_{m \in M} \sum_{u \in\left\{\varepsilon_{m} \cup \mathcal{M}_{m} \cup \mathcal{R}_{m}\right\}} x_{u, n} \Upsilon_{i, u} \Omega_{m, u} \\
&+\sum_{f_{i} \in \mathcal{F}_{i}} \sum_{u \in\left\{\mathcal{E}_{f} \cup \mathcal{M}_{f}\right\}} x_{u, n} \Upsilon_{i, u} \Omega_{m, u} \\
&+\sum_{p_{i} \in \mathcal{P}_{i}} \sum_{u \in\left\{\mathcal{E}_{p} \cup \mathcal{M}_{p}\right\}} x_{u, n} \Upsilon_{i, u} \Omega_{m, u} \\
&+\sum_{p_{i} \in \mathcal{P}_{i}} \sum_{p f_{i} \in \mathcal{P}_{i}} \sum_{u \in\left\{\varepsilon_{p f} \cup \mathcal{M}_{p f}\right\}} x_{u, n} \Upsilon_{i, u} \Omega_{m, u}
\end{aligned}
$$

In the similitude of (36) and (37), the mean traffic demand of the femto-tier is given by

$$
\varrho_{n, f, i}=\sum_{f_{i} \in \mathcal{F}_{i}} \sum_{u \in\left\{\mathcal{E}_{f} \cup \mathcal{M}_{f}\right\}} x_{u, n} \Upsilon_{i, u} \Omega_{f, u}
$$

The mean traffic load of an $S P n \in \mathcal{S}, \varrho_{n, p, i}$ in the pico-tier is given by

$$
\begin{aligned}
\varrho_{n, p, i} & =\sum_{p_{i} \in \mathcal{P}_{i}} \sum_{u \in\left\{\mathcal{E}_{p} \cup \mathcal{M}_{p}\right\}} x_{u, n} \Upsilon_{i, u} \Omega_{p, u} \\
& +\sum_{p_{i} \in \mathcal{P}_{i}} \sum_{p f_{i} \in \mathcal{P}_{\mathcal{F}_{i}}} \sum_{u \in\left\{\mathcal{E}_{p f} \cup \mathcal{M}_{p f}\right\}} x_{u, n} \Upsilon_{i, u} \Omega_{p, u}
\end{aligned}
$$

Lastly, for the clustered femto-tier, the mean traffic demand $\varrho_{n, p f, i}$ is given by

$$
\varrho_{n, p f, i}=\sum_{p_{i} \in \mathcal{P}_{i}} \sum_{p f_{i} \in \mathcal{P F}_{i}} \sum_{u \in\left\{\varepsilon_{p f} \cup \mathcal{M}_{p f}\right\}} x_{u, n} \Upsilon_{i, u} \Omega_{p f, u}
$$

\subsection{Monte Carlo method}

Numerical methods are broadly classified into two categories [86]: (1) deterministic and (2) stochastic methods. The Monte Carlo method has been widely used for randomised numerical computing owing to its simple structure and ease of implementation [87]. The Monte Carlo method is a technique for finding approximate solutions to mathematical and physical problems by the simulation of stochastic entities [88]. Alg. 3 shows the pseudo-code for the Monte Carlo method employed in this work. To solve the optimisation problem in (P1) and (P2), we employ Alg. 1 and Alg. 2. In Alg. 3, we run several thousand runs of Alg. 1 and Alg. 2 to increase the confidence levels in the total network utility.

For instance, let the outcomes of several iterations of a simulation be denoted by $\boldsymbol{C}$, such that

$$
\boldsymbol{C}=\left[\begin{array}{lllll}
c_{1} & c_{2} & c_{3} & \ldots & c_{z} \\
x_{1} & x_{2} & x_{3} & \ldots & x_{z}
\end{array}\right]
$$


where $c_{z}$ denotes the outcome of an iteration of a simulation and $x_{z}$ is the probability of occurrence for the outcome $c_{z}$. The values of probabilities $x_{1}, x_{2}, x_{3}, \cdots, x_{z}$ are such that [87]:

$$
\begin{array}{ll}
x_{o}>0, & \forall o \in Z \\
\sum_{o \in Z} x_{o}=1, & \forall o \in Z
\end{array}
$$

Therefore, the expectation of the outcomes in $\boldsymbol{C}$ is given as

$$
E(\boldsymbol{C})=\sum_{o \in Z} c_{o} x_{o}, \quad \forall o \in Z
$$

Assume that from the values obtained in $\boldsymbol{C}$, the number $q_{1}$ occurs $v_{1}$ times, the number $q_{2}$ occurs $v_{2}$ times, the number $q_{3}$ occurs $v_{3}$ times, and so on. Hence, from the laws of large numbers, we have

$$
\sum_{o \in Z} c_{o}=q_{1} v_{1}+q_{2} v_{2}+\cdots+q_{z} v_{z}
$$

Therefore,

$$
\frac{1}{Z} \sum_{o \in Z} c_{o}=q_{1} \frac{v_{1}}{Z}+q_{2} \frac{v_{2}}{Z}+\cdots+q_{z} \frac{v_{z}}{Z}
$$

With $Z$ being a large number, the frequency $\frac{v_{z}}{Z}$ of the value $q_{z}$ approaches its probability $x_{z}$ such that:

$$
\frac{v_{z}}{Z} \approx x_{z}
$$

Hence, substituting (47) into (44), we have

$$
E(\boldsymbol{C})=\frac{1}{Z} \sum_{o \in Z} c_{o}=\sum_{o \in Z} c_{o} x_{o}
$$

The expression in ((48)) is employed in the determination of the variance of $\boldsymbol{C}$ given as:

$$
\operatorname{VAR}(\boldsymbol{C})=E\left(\boldsymbol{C}^{2}\right)-(E(\boldsymbol{C}))^{2}
$$

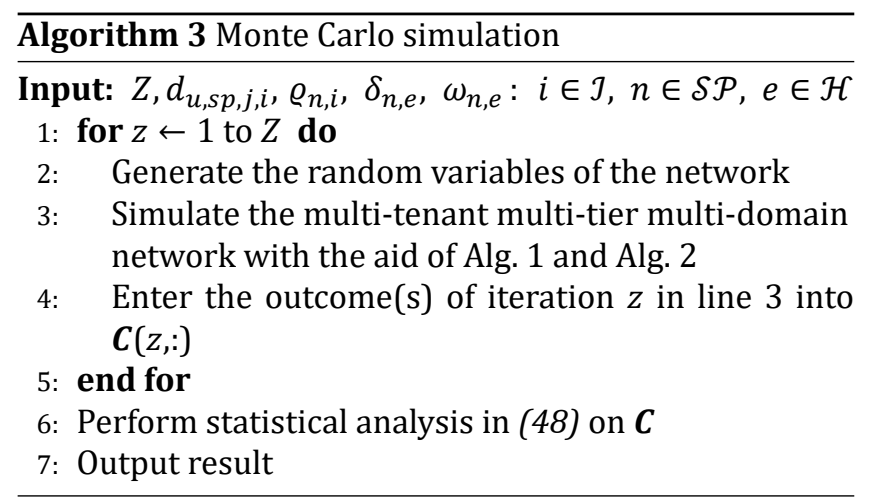

In a Monte Carlo simulation, a scenario (or an experiment) is run randomly over thousands of times. The outcomes are recorded for each iteration. The Monte Carlo result is based on the probability distribution of the several iterations.

\section{COMPLEXITY ANALYSIS}

In this section, we embark on the complexity analysis of the proposed algorithms described in Section 7. Furthermore, we approach the complexity analysis from the time complexity approach. The time complexity analysis depicts the worst-case running performance time of an algorithm. We characterise the complexity of the proposed algorithms in this work with the big- $\mathcal{O}$. The big- $\mathcal{O}$ indicates the upper-bound execution in terms of time (or memory) of an algorithm $[89,90]$. We proceed by describing the computational complexity of the multi-tier multidomain slice user association algorithms. Moreover, we discuss the computational complexity of distributed recursive backtracking for multiplayer multi-domain game algorithms, and lastly, the M-TTSD resource allocation.

\subsection{Computational complexity of the multi- tier multi-domain slice user association}

We describe the computational complexity of the multitier multi-domain slice user as $\mathcal{O}(t \cdot|\mathcal{J}|)$. Where $t$ is the number of tiers in the multi-tier network in a domain and $|\mathcal{J}|$ denotes the cardinality of the set of InP. Consequently, the computational complexity of the association algorithm is dependent on the number of tiers and domain networks.

8.2 Computational complexity of the distributed recursive backtracking multiplayer multi-domain

The complexity of the distributed recursive backtracking multiplayer multi-domain game algorithm is given by $\mathcal{O}(|\mathcal{J}| \cdot(|\mathcal{V}|+|\mathcal{S}|))$. It is important to note that in scenarios where $|\mathcal{V}| \gg|\mathcal{S}|$, its big- $\mathcal{O}$ can be approximated as $\mathcal{O}(|\mathcal{J}| \cdot|\mathcal{V}|)$. However where $|\mathcal{S}| \gg|\mathcal{V}|$, then, big- $\mathcal{O}$ is approximated as $\mathcal{O}(|\mathcal{J}| \cdot|\mathcal{S}|)$.

\subsection{Computational complexity of the multi- tier multi-tenant multi-slice multi-domain resource allocation}

In the similitude of subsections 8.1 and 8.2 , the big- $\mathcal{O}$ for the multi-tier multi-tenant multi-slice multi-domain resource allocation is given by $\mathcal{O}\left(t \cdot|\mathcal{J}| \cdot\left|\mathcal{U} \mathcal{C}_{s}\right| \cdot\left|\mathcal{U}_{s}\right|\right)$. Where $t$ denotes the number of tiers in a domain network. Additionally, the cardinalities of the set of InPs or domain networks, set of slice use-cases, set of users belonging to a SP are represented as $|\mathcal{J}|,\left|\mathcal{U} \mathcal{C}_{s}\right|$, and $\left|\mathcal{U}_{s}\right|$ respectively.

\section{NUMERICAL RESULTS}

We evaluate the performance of the M-TTSD network resource allocation scheme via extensive Monte Carlobased simulations in a MATLAB environment. We examine a 2-domain and 3-domain network with InPs having independent infrastructure and resources. Moreover, the macro-cells, picocells, femtocells, and clustered fem- 
Table 3 - Simulation parameters.

\begin{tabular}{|c|c|c|c|c|}
\hline \multicolumn{5}{|c|}{ Network Deployment } \\
\hline Network Tier & & Coverage Radius (m) & Power Budget (dBm) & No. of Cells \\
\hline Macro-cell & & 900 & 40 & 2 \\
\hline Femtocell & & 50 & 30 & {$\left[\begin{array}{lll}2 & 3 & 4\end{array}\right]$} \\
\hline Picocell & & 450 & 36.9 & {$\left[\begin{array}{lll}2 & 3 & 4\end{array}\right]$} \\
\hline Clust. Femtocell & & 50 & 30 & {$\left[\begin{array}{lll}2 & 3 & 4\end{array}\right]$} \\
\hline \multicolumn{5}{|c|}{ Network Slicing } \\
\hline Network Slice Type & Packet Arrival (Pk./s) & Packet Size bits & Packet Loss Prob. & Delay Bound (ms) \\
\hline eMBB & 20 & 9000 & $1 e^{-3}$ & 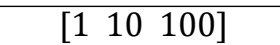 \\
\hline URLLC & 20 & 500 & {$\left[1 e^{-3} 1 e^{-4} 1 e^{-5}\right]$} & {$\left[\begin{array}{lll}1 & 10 & 100\end{array}\right]$} \\
\hline mMTC & 5 & 2000 & {$\left[1 e^{-3} 1 e^{-4} 1 e^{-5}\right]$} & {$\left[\begin{array}{lll}1 & 10 & 100\end{array}\right]$} \\
\hline
\end{tabular}

tocells have the following coverage radius of $900 \mathrm{~m}, 250 \mathrm{~m}$, $50 \mathrm{~m}$, and $50 \mathrm{~m}$, respectively. Additionally, a domain network comprises 2 femtocells; 2 picocells underlaid with 2 clustered femtocells per picocell; and a macro-cell. It is important to note that these network parameters were varied in the course of network performance evaluation. Besides, in the V2X tier, we considered a 4-lane road network with each lane having a width of $4 \mathrm{~m}$ and vehicles moving at $60 \mathrm{~km} / \mathrm{hr}$. Consequently, we assume a vehicle antenna height of $1.5 \mathrm{~m}$, vehicle noise figure of $9 \mathrm{~dB}$, and a vehicle antenna gain of $3 \mathrm{dBi}$. The network and slice usecases simulation parameters are summarised in Table 3. Furthermore, the delay bounds thresholds for the mMTC slice users can be relaxed, since it is not as stringent as the URLLC slice uses case. Moreover, the URLLC slice use case users are mobile (i.e., vehicles in the V2X-tier). Lastly, the network utility as a metric is the logarithm of the achievable rate of slice users subscribed to respective SP as indicated in (21). The metric is affected by the different network slice parameters such as the InP bandwidth, slice user average arrival rate, the delay-bound thresholds, the cell density, the coverage radius, the number of domain networks available. In the following subsections, we investigate the total utility of the network.

\subsection{Impact of the slice user intensity}

Fig. 8(a), Fig. 8(b), and Fig. 8(c) present the impact of the slice user intensity on the total network utility. With an assumed domain-network bandwidth of $400 \mathrm{MHz}$ and a small-cell density varying from 2,3 , and 4 , we examine the performance of the M-TTSD network.

Additionally, in Fig. 8(a), Fig. 8(b), and Fig. 8(c) the slice user intensity is varied from 2 to 15 to observe the network's response pattern. It is observed that as the slice user intensity increases, the total network utility increases owing to the increase in network utilisation by the increasing slice users. Besides, we observe that the proposed M-TTSD algorithm outperforms the Static-Slicing (SS) scheme [91] and GI-LARE [68] outright by an average of $20 \%$. It is seen that this value increases as the slice user intensity increases owing to resource availability from other network domains.
Similarly, in Fig. 9(a), Fig. 9(b), and Fig. 9(c), we take a close-up view of the network utility pay-off for the respective algorithms. We observe the M-TTSD network resource allocation scheme outperforms the Static-Slicing (SS) scheme [91] and GI-LARE [68].

\subsection{Impact of the network bandwidth}

In Fig. 10(a), Fig. 10(b), Fig. 10(c), and Fig. 10(d), we evaluate the performance of the network with an assumed delay bound of $10 \mathrm{~ms}$ and 100ms. In Fig. 10(a) and Fig. 10(b), we examine a 2-domain network deployment comprising 2 MVNOs and 2 SPs. We observe that the network utility of the SP increases as the domain-network bandwidth increases. Moreover, owing to the relaxation of the resource allocation QoS constraints, the network utility of the 2 SPs with a delay bound of $100 \mathrm{~ms}$ outperforms that of the $10 \mathrm{~ms}$.

In the similitude of Fig. 10(a) and Fig. 10(b), we examine the network performance in a 3-domain network deployment in Fig. 10(c) and Fig. 10(d). We observe that the utility of the network increases as the bandwidth increases. Besides, the network utility of the respective SPs in a 3domain network outperforms those of the 2-domain network owing to the availability of larger bandwidth and quite numerous access points and by extension domains available for slice user connectivity.

\subsection{Impact of the femtocell density}

Fig. 11(a) and Fig. 11(b) present the impact of the femtocell density with network parameters such as network domain bandwidth of $400 \mathrm{MHz}$, a delay bound of $100 \mathrm{~ms}$, a slice user intensity of 3 , and a packet loss probability of $10^{-5}$ on the performance of the M-TTSD network. In Fig. 11(a), we observe the impact of the femtocell density on the utility of SP1 in a 2-domain and 3-domain network, respectively. We see that the network utility increases as the femtocell density increases for both deployments, owing to efficient spectral utilisation associated with network densification. The network utility of SP1 is more evident in a 3-domain network deployment. Fig. 11(b) shows a similar trend with Fig. 11(a), herein, the utility of SP2 in a 3-domain network deployment outperforms that of a 2-domain network. 


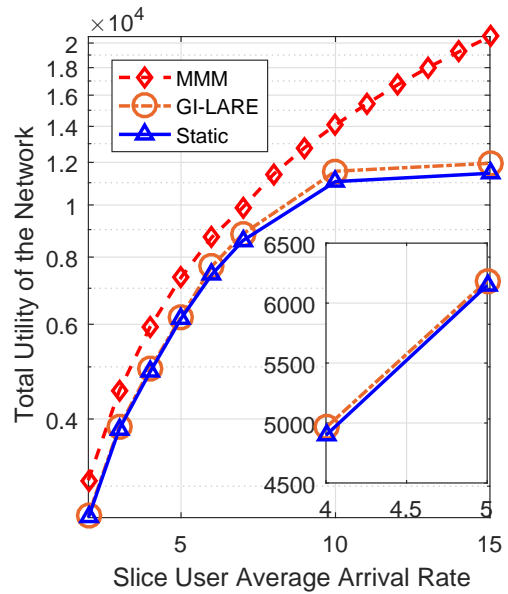

(a) With a small cell density of 2

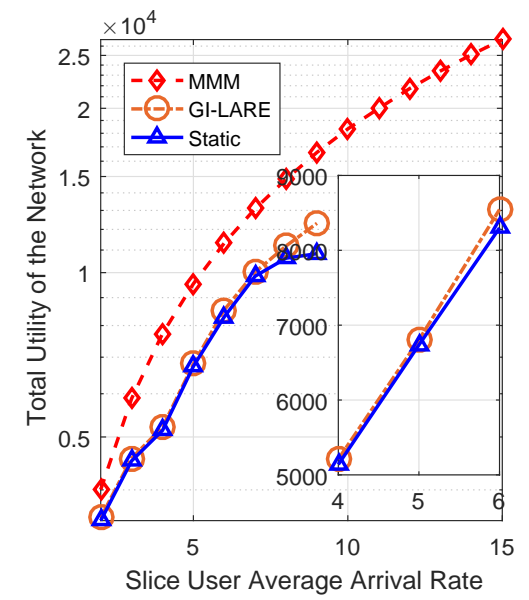

(b) With a small cell density of 3

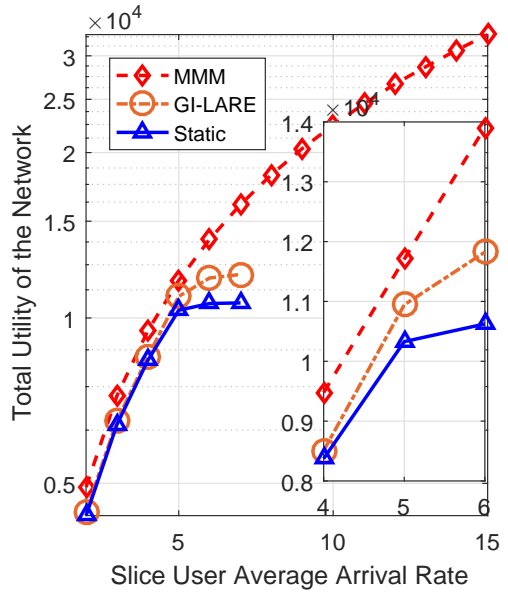

(c) With a small cell density of 4

Fig. 8 - Impact of the slice user density on the network utility.

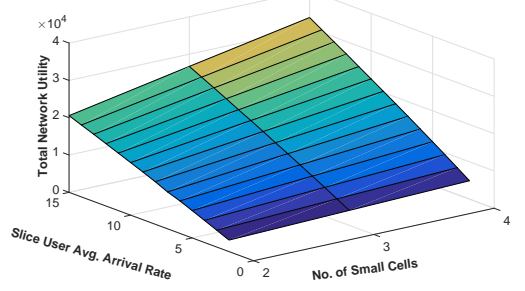

(a) Multi-Domain Multi-Tenant Multi-Slice Alg.

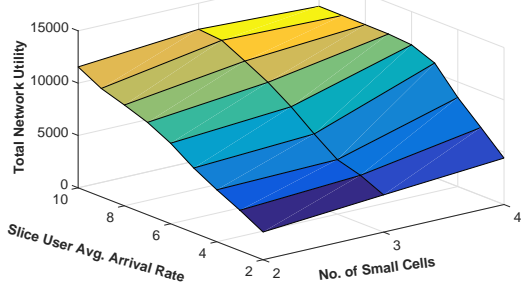

(b) GI-Lare Alg.

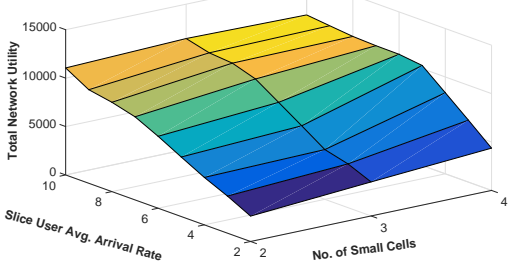

(c) Static Resource Alloc.

Fig. 9 - Impact of the slice user density on the network utility.

\subsection{Impact of the picocell density}

Fig. 12(a) and Fig. 12(b) show the effect of the picocell density with network bandwidth size, delay bound, slice user intensity, and a packet loss probability of $400 \mathrm{MHz}$, $100 \mathrm{~ms}, 3$, and $10^{-5}$ on the performance of the M-TTSD network. It is observed that as the picocell density increases, the network utility of the SPs increases. Moreover, we observe that the network utility in the 3-domain network deployment outperforms the 2-domain network for the 2 SPs, respectively, in Fig. 12(a) and Fig. 12(b).

However, comparing Fig. 11(a), Fig. 11(b), Fig. 12(a), and Fig. 12(b), we observe that the network utility for femtocell densification outperforms that of the picocell densification owing to the higher interference levels that accompany cells with higher powers, in this case, picocells. Additionally, the femtocells are much more closer to the slice users.

\subsection{Impact of the access point coverage radius}

In Fig. 13(a), Fig. 13(b), Fig. 13(c), and Fig. 13(d), we take a close-up view of the performance of M-TTSD in a 2domain and 3-domain network deployment as the coverage radius of the femtocells and picocells vary taking into consideration their peculiarities.

Fig. 13(a) and Fig. 13(b) present the impact of the coverage radius of the femtocells on the network utility in a 2-domain and 3-domain network for SP1 and SP2. With an assumed network domain bandwidth size of $400 \mathrm{MHz}$, we vary the cell radius from $10 \mathrm{~m}$ to $100 \mathrm{~m}$. To this end, we observe that the network utility dips as the cell radius increases in the 2-domain network and 3-domain network for the SP1 and SP2, respectively. Consequently, the result is due to the effect of pathloss and channel condition. Similarly, we examine the impact of the picocell coverage radius on the network utility; with a varied cell radius from $200 \mathrm{~m}$ to $450 \mathrm{~m}$ in Fig. 13(c), and Fig. 13(d). We observe the network utility for both SPs reduces as the coverage radius increases. 


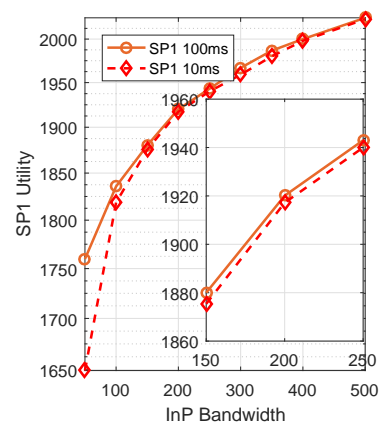

(a) SP1 in a 2-domain network.

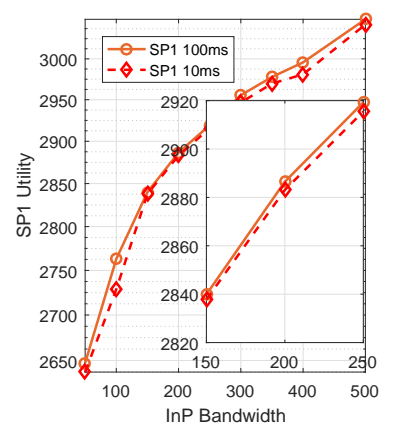

(c) SP1 in a 3-domain network.

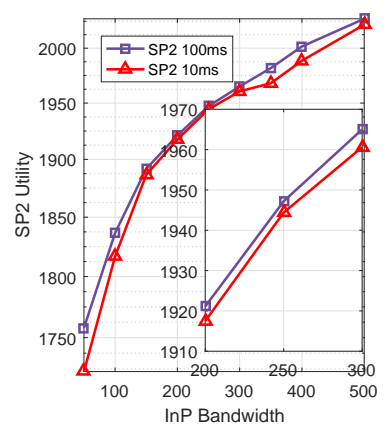

(b) SP2 in a 2-domain network.

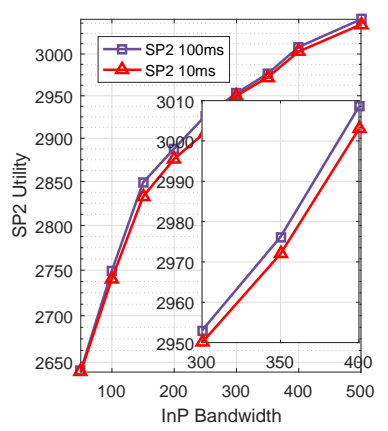

(d) SP2 in a 3-domain network.

Fig. 10 - Impact of the InP bandwidth on the utility of the SP at $10 \mathrm{~ms}$ and $100 \mathrm{~ms}$ delay bound slice requirement.
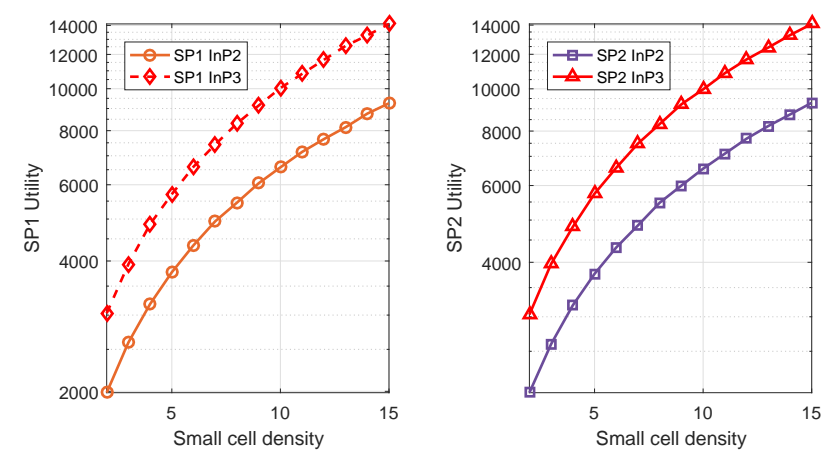

(a) SP1 in a 2 \& 3-domain network. (b) SP2 in a 2 \& 3-domain network.

Fig. 11 - Impact of the femtocell density on the utility of the SPs.

\subsection{The tier-slice ratio characterisation}

We examine the dynamic characterisation of the proposed M-TTSD algorithms with emphasis on the tier-slice ratio in the respective domains. We assume, deployment scenarios of 2 and 3-domain with bandwidth varying from $100 \mathrm{MHz}$ to $500 \mathrm{MHz}$ and maximum delay threshold set at 10ms. Fig. 14(a) and Fig. 14(b) show the dynamic characterisation of the tier-slice ratio for SP1 and SP2 in a 2-domain network.
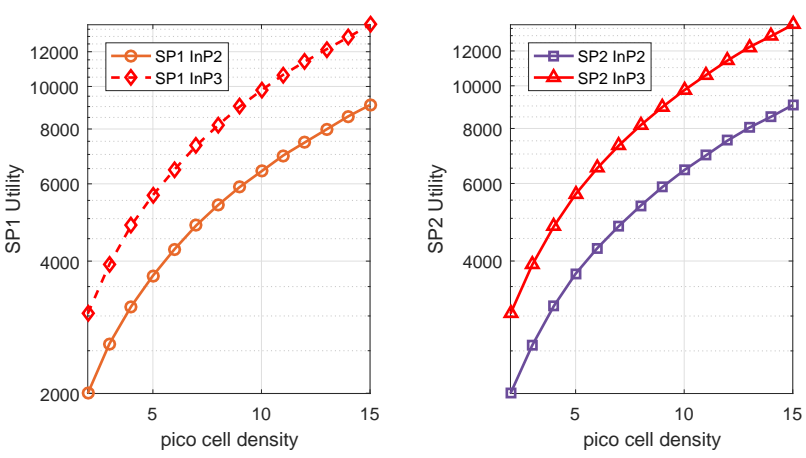

(a) SP1 in a 2 \& 3-domain network. (b) SP2 in a 2 \& 3-domain network.

Fig. 12 - Impact of the picocell density on the utility of the SPs .

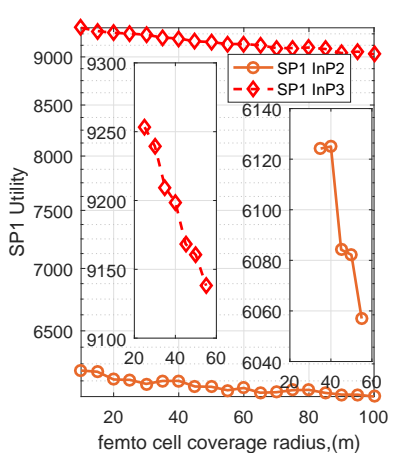

(a) SP1 in a 2-domain network.

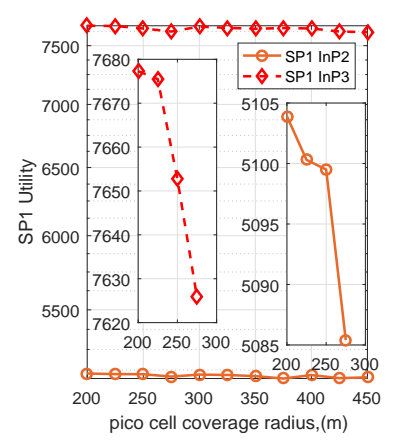

(c) SP1 in a 3-domain network.

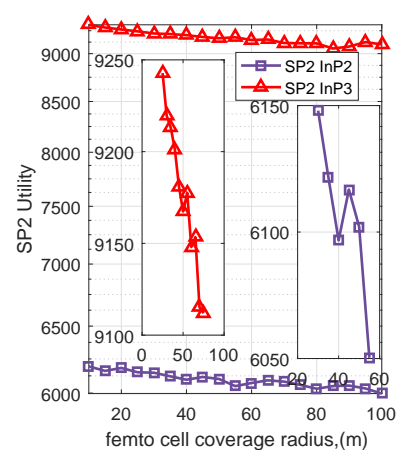

(b) SP2 in a 2-domain network.

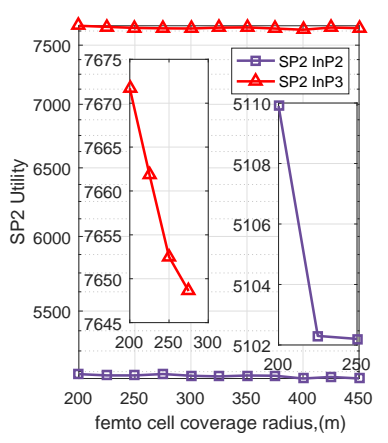

(d) SP2 in a 3-domain network.
Fig. 13 - Impact of the coverage radius of access points on the network utility in a multi-domain multi-tenant network.

We observe that in most instances, the tier-slice ratio of the clustered-femtocell is the largest. This is owing to the closeness of the femtocells to the slice users and consequently accommodating more slice users. We show the dynamic characterisation of our proposed algorithm in a 3-domain network deployment in Fig. 15(a) and Fig. 15(b). Similar to Fig. 14(a) and Fig. 14(b), the clustered-femtocells have the largest tier-slice ratio for SP1 and SP2 in all the domains.

However, the tier-slice ratio is a function of network parameters such as cell load, tier load, slice user distribution, delay bound, and packet loss probability. 


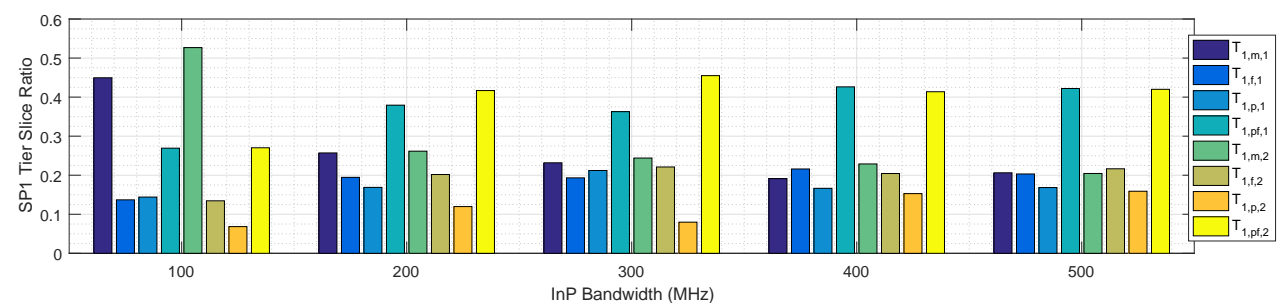

(a) SP1 tier network slice ratio $T_{1, j, i}$

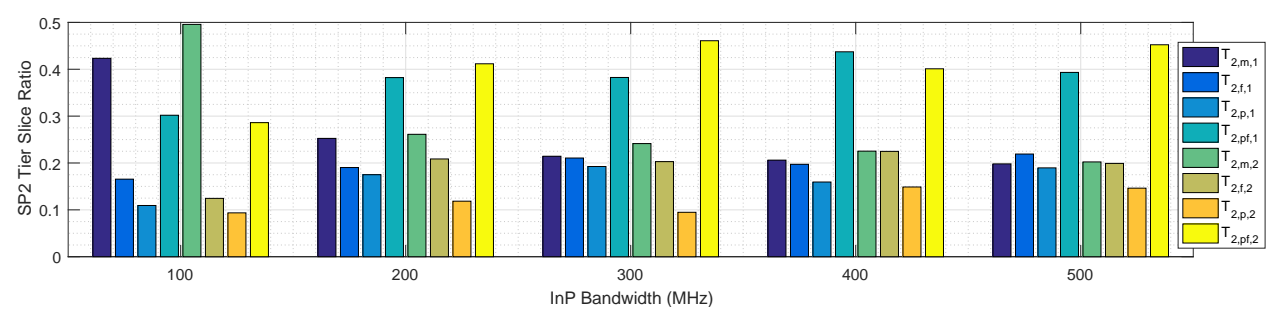

(b) SP2 tier network slice ratio $T_{2, j, i}$

Fig. 14 - Impact of the InP bandwidth on the tier network slicing ratio $T_{s, j, i}$ in a 2-domain network and a slice delay bound of $10 \mathrm{~ms}$.

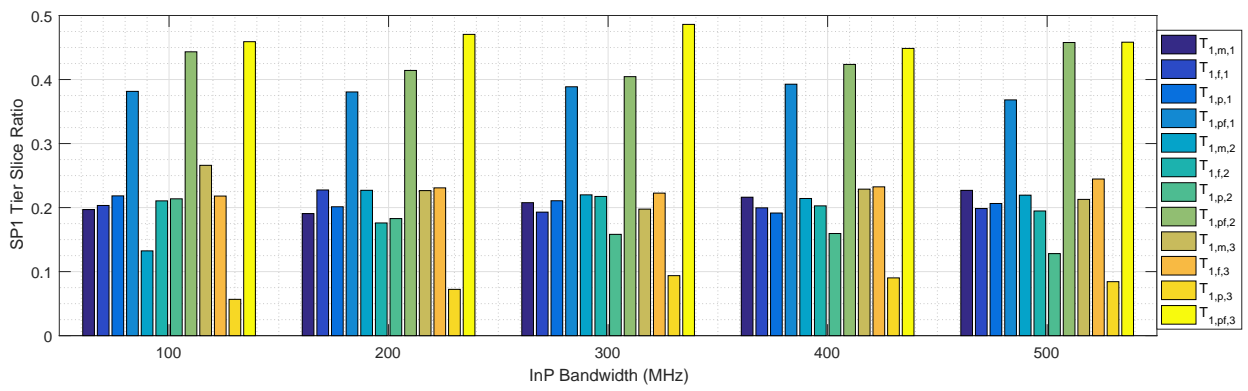

(a) SP1 tier network slice ratio $T_{1, j, i}$

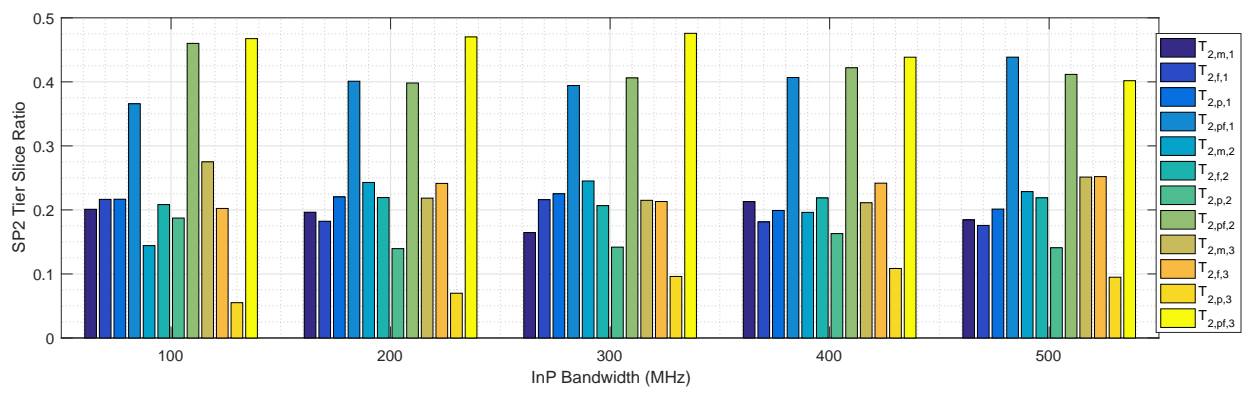

(b) SP2 tier network slice ratio $T_{2, j, i}$

Fig. 15 - Impact of the InP bandwidth on the tier network slicing ratio $T_{s, j, i}$ in a 3-domain network and a slice delay bound of $10 \mathrm{~ms}$. 
Table 4 - B2B transactions of the respective network players in a 2-domain deployment.

\begin{tabular}{|c|c|c|c|c|c|c|c|c|}
\hline Band. (MHz) & \multicolumn{2}{|c|}{ 100MHz Scenario } & \multicolumn{2}{|c|}{ 200MHz Scenario } & \multicolumn{2}{|c|}{ 300MHz Scenario } & \multicolumn{2}{|c|}{ 400MHz Scenario } \\
\hline Domain & InP 1 & InP 2 & InP 1 & InP 2 & InP 1 & InP 2 & InP 1 & InP 2 \\
\hline MVN01 bid & 437.1377 & 416.7932 & 492.0228 & 435.2518 & 461.5252 & 458.9901 & 494.4473 & 402.9160 \\
\hline MVNO2 bid & 435.8644 & 414.8111 & 451.9532 & 401.4996 & 411.4039 & 409.5608 & 439.6148 & 355.7059 \\
\hline MVN01 Res. (MHz) & 49.9198 & 49.8097 & 103.8417 & 103.8828 & 157.7897 & 157.5079 & 212.2874 & 212.4932 \\
\hline MVNO2 Res. (MHz) & 50.0802 & 50.1903 & 96.1583 & 96.1172 & 142.2103 & 142.4921 & 187.7126 & 187.5068 \\
\hline SP 1 bid for MVNO 1 & 440.3875 & 440.9423 & 440.9423 & 396.2596 & 469.4542 & 454.2560 & 458.5419 & 386.9955 \\
\hline SP 1 bid for MVNO 2 & 471.1201 & 470.9137 & 451.5686 & 417.8063 & 438.9883 & 421.4086 & 427.8220 & 363.4007 \\
\hline SP 2 bid for MVNO 1 & 487.7779 & 443.2579 & 491.5941 & 408.7465 & 426.8917 & 441.6688 & 490.5848 & 377.2100 \\
\hline SP 2 bid for MVNO 2 & 455.3364 & 418.4525 & 506.1278 & 409.3748 & 430.8421 & 450.1110 & 492.9825 & 380.7486 \\
\hline SP 1 Res. from MVNO $1(\mathrm{MHz})$ & 23.0940 & 24.5964 & 48.8744 & 52.4338 & 82.7657 & 79.6797 & 104.4882 & 108.3238 \\
\hline SP 1 Res. from MVNO $2(\mathrm{MHz})$ & 25.9951 & 27.1125 & 44.3917 & 47.6702 & 71.3611 & 70.2728 & 88.0372 & 92.0810 \\
\hline SP 2 Res. from MVNO 1 (MHz) & 26.8258 & 25.2133 & 54.9673 & 51.4490 & 75.0240 & 77.8282 & 107.7992 & 104.1694 \\
\hline SP 2 Res. from MVNO $2(\mathrm{MHz})$ & 24.0851 & 23.0778 & 51.7666 & 48.4470 & 70.8492 & 72.2193 & 99.6754 & 95.4257 \\
\hline
\end{tabular}

Table 5 - B2B transactions of the respective network players in a 3-domain deployment.

\begin{tabular}{|c|c|c|c|c|c|c|c|c|c|c|c|c|}
\hline Band. (MHz) & \multicolumn{3}{|c|}{$100 \mathrm{MHz}$ Scenario } & \multicolumn{3}{|c|}{$200 \mathrm{MHz}$ Scenario } & \multicolumn{3}{|c|}{$300 \mathrm{MHz}$ Scenario } & \multicolumn{3}{|c|}{$400 \mathrm{MHz}$ Scenario } \\
\hline Domain & InP 1 & InP 2 & InP 3 & InP 1 & InP 2 & InP 3 & InP 1 & InP 2 & InP 3 & InP 1 & InP 2 & InP 3 \\
\hline MVN01 bid & 536.7203 & 257.4576 & 413.1631 & 375.3789 & 455.6115 & 511.3260 & 350.5675 & 463.5971 & 444.5351 & 343.7390 & 409.2232 & 390.7423 \\
\hline MVNO2 bid & 595.0003 & 286.7356 & 454.6280 & 345.4384 & 410.8810 & 474.3460 & 395.7358 & 525.0341 & 506.8130 & 388.8686 & 455.4798 & 439.3983 \\
\hline MVN01 Res. (MHz) & 47.2106 & 47.2759 & 47.4116 & 105.0303 & 104.7827 & 104.6874 & 140.9176 & 140.5837 & 140.8012 & 188.0958 & 188.5488 & 188.1554 \\
\hline MVNO2 Res. (MHz) & 52.7894 & 52.7241 & 52.5884 & 94.9697 & 95.2173 & 95.3126 & 159.0824 & 159.4163 & 159.1988 & 211.9042 & 211.4512 & 211.8446 \\
\hline SP 1 bid for MVNO 1 & 598.7434 & 285.6603 & 456.8835 & 342.4516 & 415.2705 & 458.5560 & 363.4520 & 489.4903 & 458.6476 & 388.3468 & 480.0047 & 441.3709 \\
\hline SP 1 bid for MVNO 2 & 544.4724 & 257.0920 & 413.1375 & 305.9269 & 366.8423 & 414.9126 & 391.8347 & 525.3955 & 500.8746 & 349.9864 & 432.6747 & 395.2018 \\
\hline SP 2 bid for MVNO 1 & 497.4790 & 242.5254 & 387.1768 & 372.1222 & 448.5204 & 512.4984 & 338.8420 & 434.6107 & 429.3949 & 395.2414 & 445.1753 & 439.7404 \\
\hline SP 2 bid for MVNO 2 & 509.0781 & 250.2866 & 395.4414 & 323.4997 & 396.0938 & 449.1287 & 377.4907 & 488.4350 & 476.6092 & 415.5249 & 471.8150 & 475.7683 \\
\hline $\begin{array}{l}\text { SP } 1 \text { Res. from MVNO } 1 \\
(\mathrm{MHz})\end{array}$ & 25.6987 & 25.1587 & 25.4623 & 51.7523 & 51.1228 & 50.1779 & 73.2250 & 74.8409 & 73.3115 & 95.1144 & 99.2038 & 94.0071 \\
\hline $\begin{array}{l}\text { SP } 1 \text { Res. from MVNO } 2 \\
(\mathrm{MHz})\end{array}$ & 27.2110 & 26.7846 & 26.7241 & 46.0055 & 45.6403 & 45.4577 & 80.3738 & 81.7304 & 80.8016 & 97.4424 & 102.1838 & 95.8968 \\
\hline $\begin{array}{l}\text { SP } 2 \text { Res. from MVNO } 1 \\
(\mathrm{MHz})\end{array}$ & 21.5120 & 22.1172 & 21.9492 & 53.2780 & 53.6599 & 54.5095 & 67.6926 & 65.7428 & 67.4897 & 92.9815 & 89.3450 & 94.1482 \\
\hline $\begin{array}{l}\text { SP } 2 \text { Res. from MVNO } 2 \\
(\mathrm{MHz})\end{array}$ & 25.5783 & 25.9395 & 25.8643 & 48.9643 & 49.5769 & 49.8549 & 78.7086 & 77.6859 & 78.3972 & 114.4617 & 109.2674 & 115.9479 \\
\hline
\end{tabular}

\subsection{Auction bargain impact on the allocated resources to the network players at the dif- ferent hierarchies}

Table 4 and Table 5 give insight into the backtracking algorithm. We assume a delay bound of $10 \mathrm{~ms}$ and network deployment of 2 and 3-domain deployment with bandwidth varying from $100 \mathrm{MHz}$ to $400 \mathrm{MHz}$. Table 4 shows the different resource allocations for the respective MVNOs and SPs owing to their B2B transactions and bidding budget. The bidding budget is dependent on factors such as slice user distribution, delay bound requirement, and packet loss probability threshold.

\section{CONCLUSION}

In this paper, we have addressed the resource allocation problem in an M-TTSD network. We have proposed a multi-tier multi-domain slice user matching game and also a distributed backtracking multiplayer multidomain game scheme to facilitate a business-to-business resource transaction framework and to ensure maximum network utility. The maximum utility optimisation problem was transformed via the hierarchical decomposition method. The slice users were associated with access points in the respective tiers of the different domains by the concept of the multi-tier multi-domain matching game, and then network players bid for resources from higher hierarchical players via the concept of distributed multiplayer backtracking.
The backtracking scheme considered factors such as the bidder's preferences, bidding budget, cell load, tier load, and slice user distribution. The maximum utility problem is then solved using the spatial Branch-andBound (sBB) scheme.

Using the Monte Carlo simulation technique, the proposed algorithm was evaluated and compared with GI-LARE and Static Slicing (SS) resource allocation under different network deployments and parameters. The proposed algorithm outperforms these other schemes. With the 6G concept of ubiquitous wireless networks and the integration of airborne and underwater platforms to $5 G$ networks, our future work will entail investigating efficient resource allocation in network slicing from these perspectives. 


\section{APPENDIX A}

We give a detailed expression for the terms in $\left(\mathrm{P} 1^{\prime}\right)$ as follows:

$$
\begin{aligned}
& \sum_{i \in \mathcal{J}} \sum_{m \in M} \sum_{u \in\{\varepsilon \cup \mathcal{M} \cup \mathcal{R}\}} \Upsilon_{i, u} \Omega_{m, u} \boldsymbol{f}\left(\vartheta_{u, m, i}\right) \\
& =\sum_{i \in \mathcal{J}} \sum_{m \in M} \sum_{u \in \overline{\varepsilon_{m}^{\prime}}} \boldsymbol{f}\left(\vartheta_{u, m, i}\right) \\
& \overline{\mathcal{E}_{m}^{\prime}}=\left\{y \in\left(\mathcal{E}_{m} \cup \mathcal{M}_{m} \cup \mathcal{R}_{m}\right) \mid \Upsilon_{i, y} \Omega_{m, y}=1\right\} \\
& +\sum_{i \in \mathcal{J}} \sum_{f_{i} \in \mathcal{F}_{i}} \sum_{u \in \bar{\varepsilon}_{f}^{\prime}} \boldsymbol{f}\left(\vartheta_{u, m, i}\right) \\
& \overline{\varepsilon_{f}^{\prime}}=\left\{x \in\left(\varepsilon_{f} \cup \mathcal{M}_{f}\right) \mid \Upsilon_{i, x} \Omega_{m, x}=1\right\} \\
& +\sum_{i \in \mathcal{J}} \sum_{p_{i} \in \mathcal{P}_{i}} \sum_{u \in \overline{\varepsilon_{p}^{\prime}}} \boldsymbol{f}\left(\vartheta_{u, m, i}\right) \\
& \overline{\varepsilon_{p}^{\prime}}=\left\{z \in\left(\varepsilon_{p} \cup \mathcal{M}_{p}\right) \mid \Upsilon_{i, z} \Omega_{m, z}=1\right\} \\
& +\sum_{i \in \mathcal{J}} \sum_{p_{i} \in \mathcal{P}_{i}} \sum_{p f_{i} \in \mathcal{P}_{i}} \sum_{u \in \overline{\varepsilon_{p f}^{\prime}}} \boldsymbol{f}\left(\vartheta_{u, m, i}\right) \\
& \overline{\varepsilon_{p f}^{\prime}}=\left\{v \in\left(\varepsilon_{p f} \cup \mathcal{M}_{p f}\right) \mid \Upsilon_{i, v} \Omega_{m, v}=1\right\}
\end{aligned}
$$

where the first term on the RHS in (50) represents the additive utility of slice users within the macro-tier $m$ of an InP $i \in \mathcal{J}$. The second term indicates the sum utility of slice users located within a femtocell $f$ but associated with the macro-tier $m$. The third term represents the net utility of slice users located within the picocell $p$ but associated with the macro-cell $m$ of an $\operatorname{InP} i$. Lastly, the fourth term denotes the net utility of slice users associated with the macro-cell $m$, however, located in the clustered femtocell.

Similarly, from (P1'), we have (51):

$$
\begin{aligned}
\sum_{i \in \mathcal{J}} \sum_{f_{i} \in \mathcal{F}_{i}} \sum_{u \in\{\mathcal{E} \cup \mathcal{M} \cup \mathcal{R}\}} \Upsilon_{i, u} \Omega_{f, u} \boldsymbol{f}\left(\vartheta_{u, f, i}\right) & \sum_{i \in \mathcal{J}} \sum_{f_{i} \in \mathcal{F}_{i}} \boldsymbol{f}\left(\vartheta_{u, f, i}\right) \\
\overline{\mathcal{E}_{f}^{\prime \prime}}=\left\{q \in\left(\mathcal{E}_{f} \cup \mathcal{M}_{f}\right) \mid \Upsilon_{i, q}^{\prime \prime}\right. & \left.\Omega_{f, q}=1\right\}
\end{aligned}
$$

where the RHS of (51) represents the sum utility of slice users located within a femtocell $f_{i}$ and associated with that same femtocell $f_{i}$ owned by $\operatorname{InP} i$. Furthermore, we consider the third term in $\left(\mathrm{P} 1^{\prime}\right)$, and it is given as:

$$
\begin{aligned}
& \sum_{i \in \mathcal{J}} \sum_{p_{i} \in \mathcal{P}_{i}} \sum_{u \in\{\mathcal{E} \cup \mathcal{M} \cup \mathcal{R}\}} \Upsilon_{i, u} \Omega_{p, u} \boldsymbol{f}\left(\vartheta_{u, p, i}\right) \\
& =\sum_{i \in \mathcal{J}} \sum_{p_{i} \in \mathcal{P}_{i}} \sum_{\substack{u \in \overline{\mathcal{E}}_{p}^{\prime \prime} \\
\overline{\mathcal{E}_{p}^{\prime \prime}}=\left\{r \in\left(\varepsilon_{p} \cup \mathcal{M}_{p}\right) \mid \Upsilon_{i, r}\right.}} \boldsymbol{f}\left(\vartheta_{u, p, i}\right) \\
& +\sum_{i \in \mathcal{J}} \sum_{p_{i} \in \mathcal{P}_{i}} \sum_{p f_{i} \in \mathcal{P}_{i}} \sum_{\overline{u \in \overline{\varepsilon_{p f}^{\prime \prime}}}} \boldsymbol{f}\left(\vartheta_{u, p, i}\right) \\
& \overline{\varepsilon_{p f}^{\prime \prime}}=\left\{s \in\left(\varepsilon_{p f} \cup \mathcal{M}_{p f}\right) \mid \Upsilon_{i, s} \Omega_{p, s}=1\right\}
\end{aligned}
$$

where the first term on the RHS in (52) represents the net utility of slice users located within a picocell $p_{i}$ and associated with the same picocell $p_{i}$. The second term denotes the additive utility of slice users associated with the picocell $p_{i}$, but located within a clustered femtocell $p f_{i}$.

Lastly, from $\left(\mathrm{P}^{\prime}\right)$, we give a detailed expression of the fourth term as:

$$
\begin{aligned}
& \sum_{i \in \mathcal{J}} \sum_{p_{i} \in \mathcal{P}_{i}} \sum_{p f_{i} \in \mathcal{P} \mathcal{F}_{i}} \sum_{u \in\{\mathcal{E} \cup \mathcal{M} \cup \mathcal{R}\}} \Upsilon_{i, u} \Omega_{p f, u} f\left(\vartheta_{u, p f, i}\right) \\
= & \sum_{p_{i} \in \mathcal{P}_{i}} \sum_{p f_{i} \in \mathcal{P F}_{i}} \sum_{\substack{u \in \bar{\varepsilon}_{p f}^{\prime \prime} \\
\overline{\varepsilon_{p f}^{\prime \prime}}=\left\{t \in\left(\varepsilon_{p f} \cup \mathcal{M}_{p f}\right) \mid \Upsilon_{i, t}\right.}} \boldsymbol{f}\left(\vartheta_{p f, t}=1\right\}
\end{aligned}
$$

where the RHS of (53) denotes the net utility of slice users located within a clustered femtocell $p f_{i}$ and associated with same.

\section{REFERENCES}

[1] L. Velasco, L. Gifre, J. Izquierdo-Zaragoza, F. Paolucci, A. P. Vela, A. Sgambelluri, M. Ruiz, and F. Cugini. "An architecture to support autonomic slice networking". In: Journal of Lightwave Technology 36.1 (Jan. 2018), pp. 135-141. ISSN: 1558-2213. DOI: $10.1109 /$ JLT . 2017.2748233.

[2] M. Cserep, A. Recse, R. Szabo, and L. Toka. "Business network formation among $5 \mathrm{G}$ providers". In: IEEE INFOCOM 2018 - IEEE Conference on Computer Communications Workshops (INFOCOM WKSHPS). 2018, pp. 674-679.

[3] P. Camps-Arago, S. Delaere, and P. Ballon. "5G Business Models: Evolving Mobile Network Operator Roles in New Ecosystems". In: 2019 CTTE-FITCE: Smart Cities Information and Communication Technology (CTTE-FITCE). 2019, pp. 1-6. 
[4] I. Vaishnavi, J. Czentye, M. Gharbaoui, G. Giuliani, D. Haja, J. Harmatos, D. Jocha, J. Kim, B. Martini, J. MeMn, P. Monti, B. Nemeth, W. Y. Poe, A. Ramos, A. Sgambelluria, B. Sonkoly, L. Toka, F. Tusa, C. J. Bernardos, and R. Szabo. "Realizing services and slices across multiple operator domains". In: NOMS 2018 - 2018 IEEE/IFIP Network Operations and Management Symposium. 2018, pp. 1-7.

[5] T. Taleb, I. Afolabi, K. Samdanis, and F. Z. Yousaf. "On Multi-Domain Network Slicing Orchestration Architecture and Federated Resource Control". In: IEEE Network 33.5 (2019), pp. 242-252.

[6] K. Katsalis, N. Nikaein, and A. Edmonds. "MultiDomain Orchestration for NFV: Challenges and Research Directions". In: 2016 15th International Conference on Ubiquitous Computing and Communications and 2016 International Symposium on Cyberspace and Security (IUCC-CSS). 2016, pp. 189195.

[7] U. Habiba and E. Hossain. "Auction Mechanisms for Virtualization in 5G Cellular Networks: Basics, Trends, and Open Challenges". In: IEEE Communications Surveys Tutorials 20.3 (2018), pp. 22642293.

[8] S. Kuklinski, L. Tomaszewski, K. Kozłowski, and S. Pietrzyk. "Business models of network slicing". In: 2018 9th International Conference on the Network of the Future (NOF). 2018, pp. 39-43.

[9] V. Sciancalepore, C. Mannweiler, F. Z. Yousaf, P. Serrano, M. Gramaglia, J. Bradford, and I. Labrador Pavón. "A Future-Proof Architecture for Management and Orchestration of Multi-Domain NextGen Networks". In: IEEE Access 7 (2019), pp. 7921679232.

[10] S. Kuklinski, L. Tomaszewski, T. Osinski, A. Ksentini, P. A. Frangoudis, E. Cau, and M. Corici. "A reference architecture for network slicing". In: 2018 4th IEEE Conference on Network Softwarization and Workshops (NetSoft). 2018, pp. 217-221.

[11] Simina Brânzei, Yiling Chen, Xiaotie Deng, Aris Filos-Ratsikas, Søren Frederiksen, and Jie Zhang. "The fisher market game: Equilibrium and welfare". In: Proceedings of the AAAI Conference on Artificial Intelligence. Vol. 28. 1. 2014.

[12] Bharat Adsul, Ch Sobhan Babu, Jugal Garg, Ruta Mehta, and Milind Sohoni. "Nash equilibria in Fisher market". In: International Symposium on Algorithmic Game Theory. Springer. 2010, pp. 30-41.

[13] William C Brainard and Herbert E Scarf. "How to compute equilibrium prices in 1891". In: American Journal of Economics and Sociology 64.1 (2005), pp. 57-83.
[14] Li Zhang. "The efficiency and fairness of a fixed budget resource allocation game". In: International Colloquium on Automata, Languages, and Programming. Springer. 2005, pp. 485-496.

[15] Xiaohui Bei, Jugal Garg, Martin Hoefer, and Kurt Mehlhorn. "Earning and utility limits in Fisher markets". In: ACM Transactions on Economics and Computation (TEAC) 7.2 (2019), pp. 1-35.

[16] Menglan Jiang, Massimo Condoluci, and Toktam Mahmoodi. "Network slicing management \& prioritization in 5G mobile systems". In: European Wireless 2016; 22th European Wireless Conference. VDE. 2016, pp. 1-6.

[17] S. O. Oladejo and 0. E. Falowo. "5G network slicing: A multi-tenancy scenario”. In: 2017 Global Wireless Summit (GWS). Oct. 2017, pp. 88-92.

[18] S. O. Oladejo and O. E. Falowo. "Profit-Aware Resource Allocation for 5G Sliced Networks". In: 2018 European Conference on Networks and Communications (EuCNC). June 2018, pp. 43-9.

[19] Omer Narmanlioglu, Engin Zeydan, and Suayb S Arslan. "Service-aware multi-resource allocation in software-defined next generation cellular networks". In: IEEE Access 6 (2018), pp. 20348-20363.

[20] P. Caballero, A. Banchs, G. de Veciana, X. CostaPérez, and A. Azcorra. "Network Slicing for Guaranteed Rate Services: Admission Control and Resource Allocation Games". In: IEEE Transactions on Wireless Communications 17.10 (Oct. 2018), pp. 6419-6432. ISSN: 1558-2248. DOI: $10.1109 /$ TWC. 2018. 2859918.

[21] J. Zheng, P. Caballero, G. de Veciana, S. J. Baek, and A. Banchs. "Statistical Multiplexing and Traffic Shaping Games for Network Slicing". In: IEEE/ACM Transactions on Networking 26.6 (Dec. 2018), pp. 2528-2541. ISSN: 1558-2566. DOI: 10 . 1109 / TNET . 2018. 2870184.

[22] J. Zheng and P. Caballero and G. de Veciana and S. J. Baek and A. Banchs. "Statistical multiplexing and traffic shaping games for network slicing". In: 2017 15th International Symposium on Modeling and Optimization in Mobile, Ad Hoc, and Wireless Networks (WiOpt). May 2017, pp. 1-8. DoI: 10 . 23919/WIOPT. 2017.7959883.

[23] P. Caballero, A. Banchs, G. De Veciana, and X. CostaPérez. "Network Slicing Games: Enabling Customization in Multi-Tenant Mobile Networks". In: IEEE/ACM Transactions on Networking 27.2 (Apr. 2019), pp. 662-675. ISSN: 1558-2566. DOI: 10 . 1109/TNET . 2019. 2895378.

[24] Lu Ma, Xiangming Wen, Luhan Wang, Zhaoming Lu, Raymond Knopp, and Irfan Ghauri. "A Biological Model for Resource Allocation and User Dynamics in Virtualized HetNet". In: Wireless Communications and Mobile Computing 2018 (2018). 
[25] Q. Ye, W. Zhuang, S. Zhang, A. Jin, X. Shen, and X. Li. "Dynamic Radio Resource Slicing for a Two-Tier Heterogeneous Wireless Network". In: IEEE Transactions on Vehicular Technology 67.10 (Oct. 2018), pp. 9896-9910.

[26] Ying Loong Lee, Jonathan Loo, Teong Chee Chuah, and Li-Chun Wang. "Dynamic network slicing for multitenant heterogeneous cloud radio access networks". In: IEEE Transactions on Wireless Communications 17.4 (2018), pp. 2146-2161.

[27] Lei Feng, Yueqi Zi, Wenjing Li, Fanqing Zhou, Peng $\mathrm{Yu}$, and Michel Kadoch. "Dynamic Resource Allocation With RAN Slicing and Scheduling for uRLLC and eMBB Hybrid Services". In: IEEE Access 8 (2020), pp. 34538-34551.

[28] Xu Li, Rui Ni, Jun Chen, Yibo Lyu, Zhichao Rong, and Rui Du. "End-to-End Network Slicing in Radio Access Network, Transport Network and Core Network Domains". In: IEEE Access 8 (2020), pp. 29525-29537.

[29] H. Chien, Y. Lin, C. Lai, and C. Wang. "End-to-End Slicing With Optimized Communication and Computing Resource Allocation in Multi-Tenant 5G Systems". In: IEEE Transactions on Vehicular Technology 69.2 (Feb. 2020), pp. 2079-2091. ISSN: 19399359. DoI: 10.1109/TVT.2019.2959193.

[30] I. Vilà, J. Pérez-Romero, O. Sallent, and A. Umbert. "Characterization of Radio Access Network Slicing Scenarios With 5G QoS Provisioning". In: IEEE Access 8 (2020), pp. 51414-51430. ISSN: 2169-3536. DOI: 10.1109/ACCESS. 2020.2980685.

[31] Tulja Vamshi Kiran Buyakar, Harsh Agarwal, Bheemarjuna Reddy Tamma, and A Antony Franklin. "Resource Allocation with Admission Control for GBR and Delay QoS in 5G Network Slices". In: 2020 International Conference on COMmunication Systems \& NETworkS (COMSNETS). IEEE. 2020, pp. 213-220.

[32] D. Zhang, Z. Chang, F. R. Yu, X. Chen, and T. Hämäläinen. "A double auction mechanism for virtual resource allocation in SDN-based cellular network". In: 2016 IEEE 27th Annual International Symposium on Personal, Indoor, and Mobile Radio Communications (PIMRC). 2016, pp. 1-6.

[33] T. M. Ho, N. H. Tran, S. M. Ahsan Kazmi, and C. S. Hong. "Dynamic pricing for resource allocation in wireless network virtualization: A Stackelberg game approach". In: 2017 International Conference on Information Networking (ICOIN). 2017, pp. 429434.

[34] K. Zhu, Z. Cheng, B. Chen, and R. Wang. "Wireless Virtualization as a Hierarchical Combinatorial Auction: An Illustrative Example". In: 2017 IEEE Wireless Communications and Networking Conference (WCNC). 2017, pp. 1-6.
[35] S. M. A. Kazmi, N. H. Tran, T. M. Ho, and C. S. Hong. "Hierarchical Matching Game for Service Selection and Resource Purchasing in Wireless Network Virtualization". In: IEEE Communications Letters 22.1 (2018), pp. 121-124.

[36] T. LeAnh, N. H. Tran, D. T. Ngo, and C. S. Hong. "Resource Allocation for Virtualized Wireless Networks with Backhaul Constraints". In: IEEE Communications Letters 21.1 (2017), pp. 148-151.

[37] M. Robat Mili, F. Mokhtari, and F. Ashtiani. "Improving Tradeoff Among Downlink Rates of Service Providers in a VWN by Using NOMA". In: IEEE Communications Letters 23.1 (2019), pp. 156-159.

[38] Ramoni O. Adeogun. "A Novel Game Theoretic Method for Efficient Downlink Resource Allocation in Dual Band 5G Heterogeneous Network". In: Wireless Personal Communications 101.1 (2018), pp. 119-141. DOI: $10.1007 /$ s11277-018-5679-4. URL: https : //doi .org/10 . 1007/s11277-0185679-4.

[39] R. O. Adeogun. "Joint resource allocation for dual - Band heterogeneous wireless network". In: 2018 IEEE Wireless Communications and Networking Conference (WCNC). 2018, pp. 1-5.

[40] K. Thar, T. Z. Oo, Y. K. Tun, D. H. Kim, K. T. Kim, and C. S. Hong. "A Deep Learning Model Generation Framework for Virtualized Multi-Access Edge Cache Management". In: IEEE Access 7 (2019), pp. 62734-62749.

[41] Y. K. Tun, S. R. Pandey, M. Alsenwi, C. W. Zaw, and C. S. Hong. "Weighted Proportional Allocation Based Power Allocation in Wireless Network Virtualization for Future Wireless Networks". In: 2019 International Conference on Information Networking (ICOIN). 2019, pp. 284-289.

[42] Y. K. Tun, M. Alsenwi, S. R. Pandey, C. W. Zaw, and C. S. Hong. "Energy Efficient Multi-Tenant Resource Slicing in Virtualized Multi-Access Edge Computing". In: 2019 20th Asia-Pacific Network Operations and Management Symposium (APNOMS). 2019, pp. 1-4.

[43] Y. K. Tun, N. H. Tran, D. T. Ngo, S. R. Pandey, Z. Han, and C. S. Hong. "Wireless Network Slicing: Generalized Kelly Mechanism-Based Resource Allocation". In: IEEE Journal on Selected Areas in Communications 37.8 (2019), pp. 1794-1807.

[44] Y. K. Tun, A. Ndikumana, S. R. Pandey, Z. Han, and C. S. Hong. "Joint Radio Resource Allocation and Content Caching in Heterogeneous Virtualized Wireless Networks". In: IEEE Access 8 (2020), pp. 36764-36775. 
[45] B. Han, V. Sciancalepore, X. Costa-Pérez, D. Feng, and H. D. Schotten. "Multiservice-based Network Slicing Orchestration with Impatient Tenants". In: IEEE Transactions on Wireless Communications (2020), pp. 1-1.

[46] Vlastimil Křivan. "The Lotka-Volterra predatorprey model with foraging-predation risk tradeoffs". In: The American Naturalist 170.5 (2007), pp. 771-782.

[47] M. Usman, M. Qaraqe, M. R. Asghar, A. A. Gebremariam, I. S. Ansari, F. Granelli, and Q. H. Abbasi. "A Business and Legislative Perspective of V2X and Mobility Applications in 5G Networks". In: IEEE Access 8 (2020), pp. 67426-67435.

[48] Stephen 0. Ekwe, Sunday O. Oladejo, Lateef A. Akinyemi, and Neco Ventura. "A Socially-Inspired Energy-Efficient Resource Allocation Algorithm for Future Wireless Network". In: 2020 16th International Computer Engineering Conference (ICENCO). 2020, pp. 168-173. DOI: 10 . 1109/ICENC049778. 2020.9357387.

[49] Iftikhar Rasheed and Fei Hu. "Intelligent super-fast Vehicle-to-Everything 5G communications with predictive switching between mmWave and $\mathrm{THz}$ links". In: Vehicular Communications 27 (2021), p. 100303. ISSN: 2214-2096. DoI: https : // doi . org/10.1016/j.vehcom.2020.100303.

[50] Zilong Liu, Haeyoung Lee, M Omar Khyam, Jianhua He, Dirk Pesch, Klaus Moessner, Walid Saad, H Vincent Poor, et al. "6G for Vehicle-to-Everything (V2X) Communications: Enabling Technologies, Challenges, and Opportunities". In: arXiv preprint arXiv:2012.07753 (2020).

[51] Jihong Park, Sumudu Samarakoon, Hamid Shiri, Mohamed K Abdel-Aziz, Takayuki Nishio, Anis Elgabli, and Mehdi Bennis. "Extreme URLLC: Vision, challenges, and key enablers". In: arXiv preprint arXiv:2001.09683 (2020).

[52] M. K. Abdel-Aziz, S. Samarakoon, M. Bennis, and W. Saad. "Ultra-Reliable and Low-Latency Vehicular Communication: An Active Learning Approach". In: IEEE Communications Letters 24.2 (2020), pp. 367370.

[53] H. Zhou, W. Xu, J. Chen, and W. Wang. "Evolutionary V2X Technologies Toward the Internet of Vehicles: Challenges and Opportunities". In: Proceedings of the IEEE 108.2 (2020), pp. 308-323.

[54] Jian Wang, Yameng Shao, Yancong Wang, Yuming Ge, and Rundong Yu. "Physical Layer Authentication Based on Nonlinear Kalman Filter for V2X Communication". In: IEEE Access 8 (2020), pp. 163746-163757. DOI: 10.1109/ACCESS . 2020. 3022247.
[55] Monowar Hasan, Sibin Mohan, Takayuki Shimizu, and Hongsheng Lu. "Securing Vehicle-toEverything (V2X) Communication Platforms". In: IEEE Transactions on Intelligent Vehicles 5.4 (2020), pp. 693-713. DoI: 10 . 1109 / TIV . 2020 . 2987430.

[56] M. Gonzalez-Martín, M. Sepulcre, R. MolinaMasegosa, and J. Gozalvez. "Analytical Models of the Performance of C-V2X Mode 4 Vehicular Communications". In: IEEE Transactions on Vehicular Technology 68.2 (Feb. 2019). ISSN: 0018-9545. DOI: 10.1109/TVT. 2018. 2888704.

[57] Haojun Yang, Kan Zheng, Kuan Zhang, Jie Mei, and Yi Qian. "Ultra-Reliable and Low-Latency Communications for Connected Vehicles: Challenges and Solutions". In: IEEE Network 34.3 (2020), pp. 92100. DoI: 10.1109/MNET .011.1900242.

[58] G. Naik, B. Choudhury, and J. Park. "IEEE 802.11bd 5G NR V2X: Evolution of Radio Access Technologies for V2X Communications". In: IEEE Access 7 (2019), pp. 70169-70184. ISSN: 2169-3536. DoI: 10.1109/ ACCESS . 2019.2919489.

[59] V. Lakshminarasimhan and A. Knoll. "C-V2X Resource Deployment Architecture Based on Moving Network Convoys". In: 2020 IEEE 91st Vehicular Technology Conference (VTC2020-Spring). 2020, pp. 1-6.

[60] Dapeng Wu and R. Negi. "Effective capacity: a wireless link model for support of quality of service". In: IEEE Transactions on Wireless Communications 2.4 (July 2003), pp. 630-643. ISSN: 1536-1276. DoI: 10.1109/TWC. 2003.814353.

[61] Dapeng Wu and R. Negi. "Effective capacity-based quality of service measures for wireless networks". In: First International Conference on Broadband Networks. Oct. 2004, pp. 527-536. DoI: 10.1109 / BROADNETS . 2004.36.

[62] S. O. Oladejo and O. E. Falowo. "Latency-Aware Dynamic Resource Allocation Scheme for 5G Heterogeneous Network: A Network Slicing-Multitenancy Scenario". In: 2019 International Conference on Wireless and Mobile Computing, Networking and Communications (WiMob). Oct. 2019, pp. 1-7.

[63] L. Liang, G. Y. Li, and W. Xu. "Resource Allocation for D2D-Enabled Vehicular Communications". In: IEEE Transactions on Communications 65.7 (July 2017), pp. 3186-3197. DOI: 10 . 1109 / TCOMM . 2017 . 2699194.

[64] C. Guo, L. Liang, and G. Y. Li. "Resource Allocation for High-Reliability Low-Latency Vehicular Communications With Packet Retransmission". In: IEEE Transactions on Vehicular Technology 68.7 (July 2019), pp. 6219-6230. ISSN: 1939-9359. DOI: 10 . 1109/TVT. 2019. 2919181. 
[65] NGMN. "Winner II Channel Models, Standard IST4-027756 WINNER II D1.1.2 v1.2". In: Accessed on: 2019-05-18. Sept., 2007. URL: https : / /www . cept .org/files/8339/winner $2 \% 20-\% 20$ final $\%$ 20report.pdf.

[66] Y. Zhang, C. Lee, D. Niyato, and P. Wang. "Auction Approaches for Resource Allocation in Wireless Systems: A Survey". In: IEEE Communications Surveys Tutorials 15.3 (2013), pp. 1020-1041.

[67] R. A. Berry and R. Johari. Economic Modeling in Networking: A Primer. Now Foundations and Trends, 2013. DOI: $10.1561 / 1300000011$.

[68] S. O. Oladejo and O. E. Falowo. "Latency-Aware Dynamic Resource Allocation Scheme for Multi-Tier 5G Network: A Network Slicing-Multitenancy Scenario". In: IEEE Access 8 (2020), pp. 74834-74852.

[69] M. Bennis, M. Debbah, and H. V. Poor. "Ultrareliable and Low-Latency Wireless Communication: Tail, Risk, and Scale". In: Proceedings of the IEEE 106.10 (2018), pp. 1834-1853.

[70] Janko Gravner. Lecture notes for Introduction Probability. Dec. 2017.

[71] A. Sinha and E. Modiano. "Network utility maximization with heterogeneous traffic flows". In: 2018 16th International Symposium on Modeling and Optimization in Mobile, Ad Hoc, and Wireless Networks (WiOpt). 2018, pp. 1-8.

[72] S. Lamparter, D. Oberle, and A. Eberhart. "Approximating service utility from policies and value function patterns". In: Sixth IEEE International Workshop on Policies for Distributed Systems and Networks (POLICY'05). 2005, pp. 159-168.

[73] Ralph L Keeney, Howard Raiffa, et al. Decisions with multiple objectives: preferences and value tradeoffs. Cambridge university press, 1993.

[74] D. P. Palomar and M. Chiang. "A Tutorial on Decomposition Methods for Network Utility Maximazation". In: IEEE Journal on Sel. Areas in Comms. (JSAC) 24.8 (2006), pp. 1439-1451.

[75] Jame Reilly, Magda Chatzaki, and Alex Galis. "Quality of Service and Routing in Multi-Domain Broadband Transport Networks". In: Multi-domain communication management systems. Ed. by Alex Galis. Boca Raton, FL., USA: CRC Press, 2000. Chap. 5, pp. 49-78.

[76] Z. Mlika, M. Goonewardena, W. Ajib, and H. Elbiaze. "User-Base-Station Association in HetSNets: Complexity and Efficient Algorithms". In: IEEE Transactions on Vehicular Technology 66.2 (Feb. 2017), pp. 1484-1495. DOI: 10 . 1109 / TVT . 2016 . 2558501.
[77] Z. Mlika, E. Driouch, and W. Ajib. "User Association Under SINR Constraints in HetNets: Upper Bound and NP-Hardness". In: IEEE Communications Letters 22.8 (Aug. 2018), pp. 1672-1675. DoI: 10 . 1109/LCOMM. 2018. 2840714.

[78] T. Hoessler, P. Schulz, E. A. Jorswieck, M. Simsek, and G. P. Fettweis. "Stable Matching for Wireless URLLC in Multi-Cellular, Multi-User Systems". In: IEEE Transactions on Communications 68.8 (2020), pp. 5228-5241. DOI: 10 . 1109 / TCOMM . 2020 . 2995150.

[79] Y. Gu, W. Saad, M. Bennis, M. Debbah, and Z. Han. "Matching theory for future wireless networks: fundamentals and applications". In: IEEE Communications Magazine 53.5 (May 2015), pp. 52-59. DoI: 10.1109/MCOM. 2015.7105641.

[80] Youssef Hamadi, Christian Bessiere, and Joël Quinqueton. "Backtracking in distributed constraint networks". In: Proceedings ECAI'98. Citeseer. 1998, pp. 219-223.

[81] Youssef Hamadi and Georg Ringwelski. "Boosting distributed constraint satisfaction". In: Journal of Heuristics 17.3 (2011), pp. 251-279.

[82] Grzegorz Kondrak and Peter Van Beek. "A theoretical evaluation of selected backtracking algorithms". In: Artificial Intelligence 89.1-2 (1997), pp. 365387.

[83] Solomon W Golomb and Leonard D Baumert. "Backtrack programming". In: Journal of the ACM (JACM) 12.4 (1965), pp. 516-524.

[84] Vipin Kumar. "Algorithms for constraintsatisfaction problems: A survey". In: AI magazine 13.1 (1992), pp. 32-32.

[85] John DC Little. "A proof for the queuing formula: $\mathrm{L}=\lambda$ W". In: Operations research 9.3 (1961), pp. 383-387.

[86] J. Chen and L. Feng. "Using Lower and Upper Bounds to Increase the Computing Accuracy of Monte Carlo Method". In: 2010 International Conference on Computational and Information Sciences. 2010, pp. 630-633.

[87] Ilya M Sobol. A primer for the Monte Carlo method. CRC press, 1994.

[88] R. M. Karp and M. Luby. "Monte-Carlo algorithms for enumeration and reliability problems". In: 24 th Annual Symposium on Foundations of Computer Science (sfcs 1983). 1983, pp. 56-64.

[89] Donald E Knuth. "Big omicron and big omega and big theta". In: ACM Sigact News 8.2 (1976), pp. 18-24.

[90] PE Black. "big-O notation, Dictionary of algorithms and data structures". In: US National Institute of Standards and Technology (2008). 
[91] X. Costa-Perez, J. Swetina, T. Guo, R. Mahindra, and S. Rangarajan. "Radio access network virtualization for future mobile carrier networks". In: IEEE Communications Magazine 51.7 (July 2013), pp. 27-35. ISSN: 1558-1896. DOI: 10 .1109/MCOM . 2013.6553675.

\section{AUTHORS}

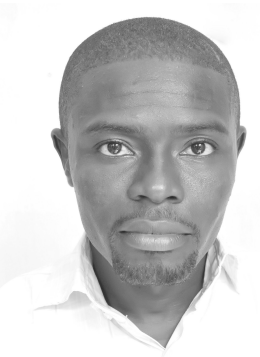

Sunday 0. Oladejo received a B.Eng degree in electrical and electronic engineering from the Federal University of Technology, Akure, Nigeria, in 2004 and an M.Eng degree in communication engineering from the Federal University of Technology, Minna, Nigeria, in 2016. He is currently pursuing a $\mathrm{PhD}$ degree in electrical engineering at the University of Cape Town, South Africa. From 2007 to 2017, he was Senior Core Network Engineer with Glo-Mobile, Nigeria. His research interest includes radio resource management in wireless networks, and artificial intelligence.

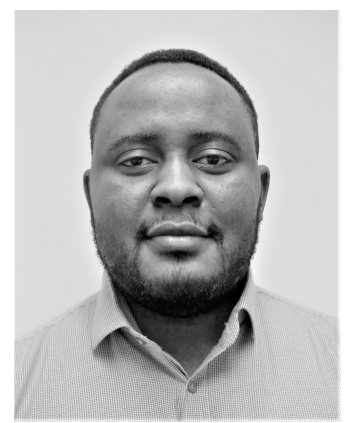

Stephen 0. Ekwe received a B.Eng degree in electrical and electronic engineering from the Cross River University of Technology, Calabar, Nigeria, in 2008 and an M.Sc. degree in Personal, Mobile and Satellite Communication from the University of Bradford, West Yorkshire, England, in 2013. He is currently a doctoral candidate in electrical engineering at the University of Cape Town, South Africa. His research interest includes $5 \mathrm{G}$, Internet of Things, social network analytics, machine learning, and resource optimization and management in wireless communication networks.

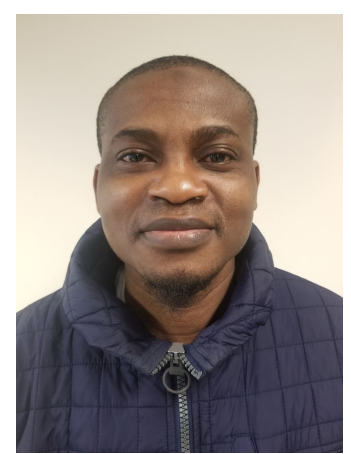

Lateef A. Akinyemi is a lecturer and researcher of electronic and computer engineering at the Department of Electronic and Computer, Faculty of Engineering, Lagos State University, Lagos, Nigeria. He obtained a B.Sc. (First Class Hons.) Electronic and Computer Engineering (Computational Electronics) at Lagos State University, Lagos, Nigeria, a Master of Science in electronic and computer engineering at Lagos State University, Lagos, Nigeria and Master of Science in electrical and electronics engineering (Communication Engineering Option), University of Lagos, Akoka, Nigeria, and $\mathrm{PhD}$ in electrical engineering, Department of Electrical Engineering, Faculty of Engineering and the Built Environment, University of Cape town, Western Cape, South Africa. His research areas are wireless communications and its applications, computational electronics and analysis, wavelets and frames, electromagnetic fields and waves, numerical techniques (SFDM, CFDM, DFT), modelling and simulations of quantum-inspired nanoparticles and devices, microwave engineering and antennas. 